\title{
Towards realistic non-linear receding-horizon spectral control of wave energy converters
}

\author{
Alexis Mérigaud*, John V. Ringwood \\ Centre for Ocean Energy Research, Maynooth University, Maynooth, Ireland
}

\section{A R T I C L E I N F O}

\section{Keywords:}

Wave energy converters

Power maximisation

Optimal control

Receding-horizon control

Fourier spectral control

Constraints

Non-linear systems

Trajectory tracking

Extended Kalman filter

Measurement noise

Gaussian process

\begin{abstract}
A B S T R A C T
Non-linear, power-maximising control of wave energy converters (WECs) can be achieved within a recedinghorizon control framework, whereby an upper loop calculates a reference trajectory in real-time, ensuring maximal power absorption under operational constraints, while a tracking loop drives the device along the generated trajectory. This paper articulates the four fundamental components of such a control strategy: reference generation calculations, tracking loop, and wave excitation estimation and forecasting. The upper-loop optimisation problem is efficiently solved through a Fourier spectral method, taking into account non-linear dynamics and constraints. Tracking is achieved through a linear state feedback, combined with a non-linear feed-forward term. An extended Kalman filter is used for excitation force estimation, based on noisy WEC position and acceleration measurements. Finally, wave excitation forecasts are based on a linear predictor, whose coefficients are derived from the wave spectrum (on a sea-state-by-sea-state basis). The practical issues and trade-offs, which arise when the four components listed above are combined within a practical implementation, are investigated by means of realistic numerical simulations, using a WEC model comprising a combination of static and velocity-dependent non-linear forces.
\end{abstract}

\section{Introduction}

Power-maximising control has the potential to significantly improve the economic competitiveness of WECs (Ringwood, Bacelli, \& Fusco, 2014). However, the practical implementation of real-time WEC control faces significant technical barriers, including the following:

- Due, in particular, to radiation force memory effects, the optimal control law for WEC power maximisation is, in general, non-causal, i.e. the knowledge of future wave excitation is required (Falnes, 2002);

- As stressed in Penalba Retes, Mérigaud, Gilloteaux, and Ringwood (2015), hydrodynamic non-linearities tend to be highlighted under actively controlled conditions compared to, for example, passive linear damping. In addition, non-linear dynamics may also stem from the characteristics of the power take-off (PTO) machinery or from other physical components, such as the mooring system. Therefore, a realistic WEC control system should be able to accommodate non-linear effects where appropriate;

- Operational constraints must be taken into account, to prevent the WEC or PTO system from exceeding its physical limitations.

Receding-horizon control provides a relevant framework to address these challenges, via the following characteristics:
- Taking into account wave excitation forecasts over a finite time horizon, the optimal control force or WEC trajectory is calculated in real time, and updated as new wave input forecasts become available (Gieske, 2007);

- The optimal control force or trajectory calculation, which is in essence an optimisation problem, can take into account non-linear WEC dynamics and operational constraints.

The general receding-horizon WEC control philosophy is illustrated in Fig. 1, showing the reference WEC velocity (optimal velocity prediction) updated at two consecutive time steps. The true optimal velocity is the one which would maximise power absorption, if the true wave excitation signal was perfectly known over an infinite time horizon. As illustrated in the figure, the optimal velocity, which is calculated within a finitehorizon window, differs from the true optimal velocity.

Due to the consecutive updates of the reference trajectory or control input, a receding-horizon control scheme involves sequential use of an efficient optimisation algorithm. Regardless of whether such an algorithm generates a reference trajectory, control force, or both, it will be termed 'reference generator' (RG).

Receding-horizon WEC control strategies are reviewed in Faedo, Olaya, and Ringwood (2017). The majority of studies use linear or nonlinear model predictive control (MPC) as a RG, where the variables

\footnotetext{
* Corresponding author.

E-mail address: alexis.merigaud.2015@mumail.ie (A. Mérigaud).
} 


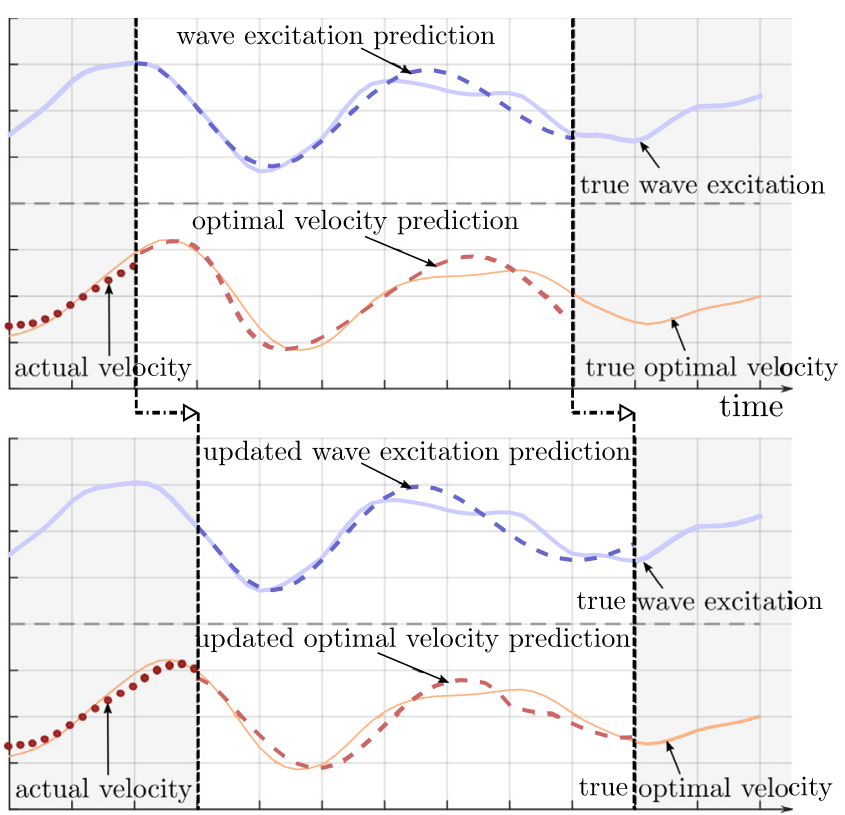

Fig. 1. Receding-horizon WEC control philosophy - optimal velocity trajectory updates at two consecutive time steps. Solid blue (resp. orange): true wave excitation (resp. true optimal WEC trajectory). Dashed blue (resp. red): predicted wave excitation (resp. predicted optimal WEC trajectory). Dotted red: actual trajectory followed by the WEC (trying to track the predicted optimal trajectory).

(state variables, control input) are discretised in time, and the RG yields a sequence of control inputs over the receding time window. The computational difficulties associated with a real-time implementation of MPC are highlighted by a number of authors (for example Li, Weiss, Mueller, Townley, \& Belmont, 2012, Richter, Magana, Sawodny, \& Brekken, 2013, Tona, Nguyen, Sabiron, \& Creff, 2015), and tend to reduce the time-horizon which can be effectively used as a receding window length.

Alternatively, recent years have witnessed the development of spectral (S) and pseudo-spectral (PS) techniques for WEC control applications (Faedo et al., 2017) which, instead of resorting to a time discretisation, describe the optimisation variables using sets of basis functions of various kinds. Fig. 2 shows several examples of such basis functions, in comparison to the (more usual) time discretisation (i.e. zero-order hold, or $\mathrm{ZOH}$, in the figure), for the approximation of a signal $f$ which could be, for example, the wave excitation force contained within the receding window. As can be seen in Fig. 2b, all other methods require less basis functions than $\mathrm{ZOH}$ for the same level of signal fidelity. This is a well-known property of spectral methods: for a sufficiently smooth target function, the accuracy of the spectral approximation improves more than linearly with the number of basis functions (Boyd, 2001).

$S$ and PS methods have shown some promise in efficiently solving the WEC control problem (Bacelli, Genest, \& Ringwood, 2015; Bacelli \& Ringwood, 2014; Genest \& Ringwood, 2017; Li, 2015; Mérigaud \& Ringwood, 0000a, 2017). In addition to the computational benefits resulting from a potentially smaller number of variables involved in the RG optimisation (since, as seen in Fig. 2b, less functions are required to accurately describe input signals and variables), spectral and pseudospectral techniques also provide a natural way to modulate the degree of smoothness of reference trajectories or control inputs.

In particular, assuming a Fourier spectral control (FSC) formulation - i.e. using harmonic sinusoids as a functional basis - (Mérigaud \& Ringwood, 0000a) details how the solution speed of the FSC problem can be significantly improved by explicit computation of the gradient and Hessian of the objective function. However, such a functional basis assumes periodicity of the wave input, while the finite-length wave excitation signal contained in the receding window is, in general, non-periodic

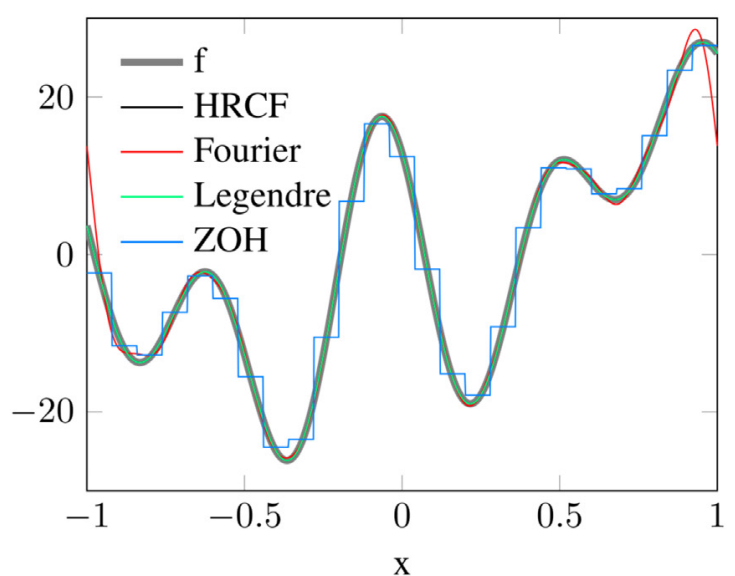

(a)

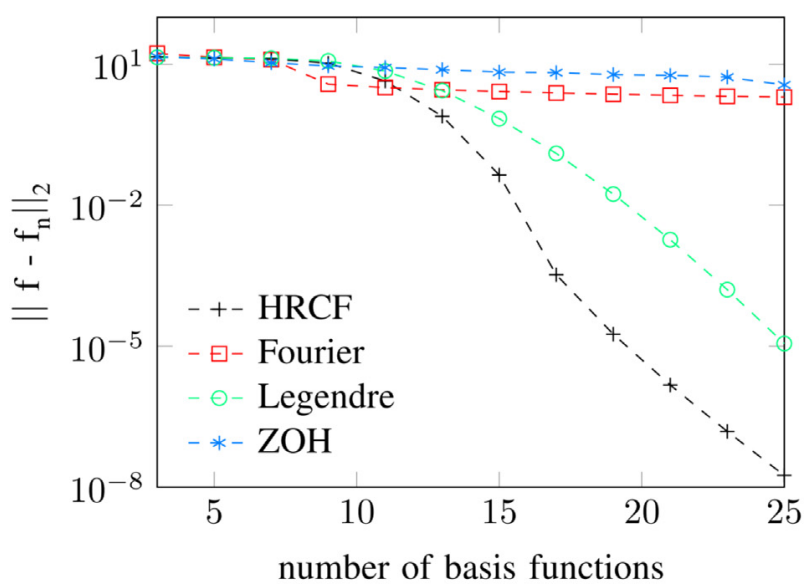

(b)

Fig. 2. (a) shows the approximation of a signal $f$ using different sets of orthogonal functions - reproduced from (Genest \& Ringwood, 2017). (b) shows the approximation error as a function of the number of basis functions. HRCF: half-range Chebyshev Fourier basis functions; Fourier: Fourier basis functions; Legendre: Legendre polynomials; ZOH: zero-order hold.

(in the example of Fig. 2, the Fourier basis yields larger approximation errors than HRCF and Legendre polynomials). Nevertheless, applying a windowing function to the finite-length wave excitation signal, spanned by the receding horizon, prior to the corresponding control calculation, can make the Fourier description appropriate (Auger, Mérigaud, \& Ringwood, 0000). In this paper, a FSC solution method, applied to the windowed wave signal, is used as the RG optimisation algorithm. More detail is given in Section 3 about the FSC solution technique, and its practical implementation in a receding-horizon fashion.

In a receding-horizon WEC control implementation, as mentioned above, the RG calculations may directly provide the required control input (Faedo et al., 2017) (typically, the PTO force). Alternatively, the RG may compute a reference WEC trajectory (in terms of WEC position and/or velocity), which is subsequently followed by means of a tracking loop (TL), making use of feedback control (Fusco \& Ringwood, 2014). The latter indirect approach could offer several potential benefits:

- It has been highlighted in Mérigaud and Ringwood (2017) and Nielsen, Zhou, Kramer, Basu, and Zhang (2013) that, under some conditions, calculations of the optimal WEC trajectory are independent of inertial terms and (linear or non-linear) static forces. Therefore, RG calculations naturally exhibit robustness to modelling errors in inertial and static terms, and, by ignoring such modelling terms where appropriate, may be made more efficient. 
- The TL can make use of well-known tools from linear or nonlinear feedback control, to ensure good tracking of the reference WEC motions, and possibly robustness to modelling errors (Fusco \& Ringwood, 2014).

In contrast, computing the optimal control force directly requires accurate modelling of all the WEC dynamics (Mérigaud \& Ringwood, 2017).

However, in reality, the actual control force for trajectory tracking may consist of some combination of feed-forward and feedback-like terms, such as in the back-stepping procedure used in Davidson, Genest, and Ringwood (2017). Conversely, in MPC approaches, where the RG computes the control input, the current state of the device (in terms of position and/or velocity) is generally taken into account in the RG optimisation problem, which implicitly introduces a form of feedback effect into the control law. In the control framework proposed in this paper, the RG primarily computes a reference WEC trajectory but, regarding the trajectory tracking, a simple state feedback term is combined with a feed-forward control input (also resulting from the RG optimisation), aimed at mitigating the task of the reactive control action.

Regardless of the RG calculation method, estimates and forecasts of the wave excitation signal are necessary. Nevertheless, the issue of wave force estimation (WE) and forecasting (WF), and their interaction with control performance, are rarely addressed in receding-horizon WEC control studies, where the wave force is generally assumed to be perfectly known over the receding horizon considered.

A few exceptions can be found in Andersen, Pedersen, Nielsen, and Vidal (2015), Brekken (2011), Cavaglieri, Bewley, and Previsic (2015), Hals, Falnes, and Moan (2011), Li et al. (2012), Tona et al. (2015) and de la Villa Jaén, Santana, et al. (2014), where WF is explicitly taken into account. In Li et al. (2012), and other works by the same authors, as well as in Cavaglieri et al. (2015), the WF technique assumes availability of measurement equipment, measuring the wave field surrounding the WEC. But, in most cases, Andersen et al. (2015), Brekken (2011), Hals et al. (2011), Tona et al. (2015), and de la Villa Jaén et al. (2014), WF is carried out assuming perfect measurements of the past excitation values, extrapolated into the future using some time-series regression technique. In particular, auto-regressive (AR) models are used by several authors (Brekken, 2011; Tona et al., 2015; de la Villa Jaén et al., 2014). The issue of the optimisation of the AR coefficients, at regular intervals, is identified as a subject for investigation (Brekken, 2011; Tona et al., 2015). Finally, WF has been addressed in more detail in Fusco and Ringwood (2010), independently from its use within real-time control.

Recent work (Mérigaud \& Ringwood, 0000b) shows that, assuming stationary, Gaussian waves, and for a given measurement configuration, i.e. for a given set of measurement instants and locations, relative to the instants and locations at which the variable (wave elevation or excitation force) is predicted:

- the optimal predictor is linear, and

- its coefficients can be directly identified from the wave spectrum (and thus should only be updated as the wave condition evolves, e.g. every half-hour).

Therefore the technique used in Mérigaud and Ringwood (0000b), retained in this study (see Section 6), and thereafter termed spectrumbased predictor (SBP), simply assumes that the wave spectrum is known, and requires no data-based identification procedure. For this paper, no measurements are assumed in the vicinity of the WEC; instead, only past values of the estimated excitation force are used for the forecasts.

WE may be carried out through measurements of physical quantities, directly related to the incoming wave force, such as the pressure distribution along the WEC hull, using a number of pressure sensors (Abdelkhalik, Zou, Bacelli, Robinett, Wilson, \& Coe, 2016), or up-wave freesurface elevation measurements (Cavaglieri et al., 2015; Guo, Patton, \& Jin, 2017). Alternatively, the excitation force can be estimated, solely based on measurements of WEC dynamics, which are usually available via the WEC control system (Peña-Sanchez, Garcia-Abril, Paparella, \&
Ringwood, 2018). However, the interaction between real-time control and WE has to be investigated in detail (although WE is carried out in-line in Brekken (2011), no measurement noise or modelling uncertainties are considered). Therefore, in this paper, and based on somewhat minimal assumptions on available measurement equipment, the excitation force is estimated through an Extended Kalman Filter (EKF), based solely on measured WEC dynamics, namely position and acceleration (which, in practice, would be obtained using a position sensor and an inertial measurement unit, respectively). The effect of measurement noise level is also investigated.

Overall, the paper aims to answer the following question: With rather pessimistic assumptions regarding the problem complexity (nonlinear dynamics, linear and non-linear constraints) and available measurements (only noisy position and acceleration measurements are available), and with a realistic receding-horizon set-up articulating the four components (WE, WF, RG, TL), what can reasonably be achieved using state-of-the art control calculation techniques? Are the results far from the true optimal power, which would be achieved in idealised conditions, i.e. if the excitation force was known for an infinite time horizon and if the device was able to perfectly track the true optimal trajectory?

Finally, the design components of the control architecture presented in this paper are pictured in Fig. 3, without detailed specification of the techniques chosen for each task (RG, TL, WE and WF). The specific choices made concerning each of the four components are discussed in more detail in the corresponding sections indicated in Fig. 3. The authors believe that the proposed framework is reasonably general, and that various techniques can be investigated in future work, to replace some of the specific choices presented in this paper. More importantly than the techniques chosen, the interactions between RG, TL, WE and $\mathrm{WF}$ are the main focus of this paper.

The rest of this paper is organised as follows: Section 2 describes the general class of WEC models considered in this study. Sections 3 to 6 detail the specific solutions chosen for RG calculations, TL, WE and WF. Section 7 describes the WEC model and numerical set-up developed for the practical assessment of the proposed control framework. The corresponding numerical results are given in Section 8, showing the interplay between the four components of the control structure. Finally, conclusions and directions for future work are given in Section 9.

\section{WEC dynamical model}

Consider a 1-DoF WEC, whose generalised position is described by means of a scalar coordinate $z$. Assume that Newton's second law, describing the WEC dynamics, can be written in the following form:

$m \ddot{z}=l(z, \dot{z})+n(z, \dot{z})+u(t)+e(t)$

where:

- $m \ddot{z}$ represents the system inertia (possibly, taking into account the radiation infinite-frequency added mass);

- $l(z, \dot{z})$ represents forces which depend on $z$ and $\dot{z}$ in a linear way. For example, if all the hydrodynamic forces acting on the device are linearly modelled, $l$ consists of the terms of the well-known Cummins' equation (Cummins, 1962):

$l(z, \dot{z})=\int_{-\infty}^{t} k_{r}(t-\tau) \dot{z}(\tau) d \tau+s_{h} z$

where $s_{h}$ is a hydrostatic stiffness coefficient, and the radiation force memory terms are computed as a convolution product between the past values of the velocity and the radiation impulse response function $k_{r}$;

- $n(z, \dot{z})$ is an analytical expression, containing the forces which are non-linearly modelled as a function of $z$ and $\dot{z}$. Obviously, if a part of the hydrodynamic force (for example, the hydrostatic restoring term) is non-linearly modelled within $n$, the corresponding terms have to be removed from $l$ in Eq. (2); 


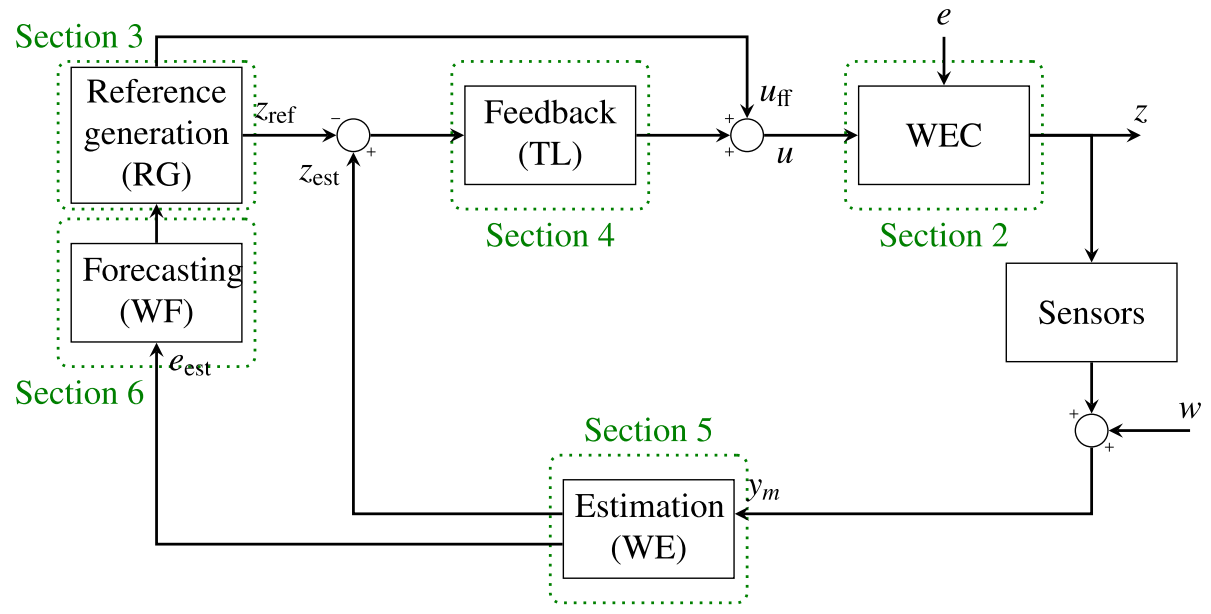

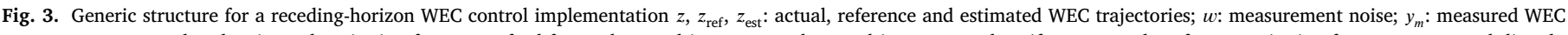

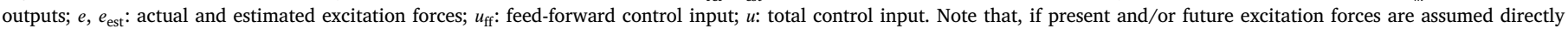
available, WF and/or WE are not needed.

- The control input $u(t)$ is the force exerted by the PTO system on the WEC;

- $e(t)$ is an additive wave excitation term, which generally consists of the linear wave excitation or diffraction force.

Note that the formulation in Eq. (1), and the mathematical developments in Section 3, can be straightforwardly extended to more general types of non-linear effects, of the form $n(z, \dot{z}, \ddot{z}, t)$, where the dependence on $t$ allows for modelling a non-linear relationship between the forces and a wave input signal (e.g. non-linearities taking into account the free-surface elevation at the device location). However, for the sake of clarity and conciseness, such a possibility is not further pursued in this paper.

\section{Receding-horizon control calculations using Fourier basis func- tions}

The performance of the proposed control structure heavily relies on the quality of RG calculations (i.e. how close the reference trajectory evaluated by the RG algorithm is to the actual optimal trajectory), and on the RG computational efficiency, in particular in the presence of non-linear forces and constraints (if the RG optimisation cannot be carried out fast enough, no real-time implementation can be reasonably considered). Therefore, RG calculations can be seen as the core of the proposed receding-horizon control structure.

The technique retained in this work is based on a FSC method (Mérigaud \& Ringwood, 0000a) whereby, assuming a periodic wave input, the steady-state optimal control problem is solved through a projection of the wave input and optimisation variables onto a Fourier basis. Non-linear forces, as well as linear or non-linear constraints can be considered within the FSC formulation, with high efficiency (Mérigaud \& Ringwood, 0000a).

The FSC method is now presented, along with its articulation in a receding-horizon fashion.

\subsection{Fourier spectral formulation of the unconstrained control problem}

The optimal, power-maximising control problem is considered for a periodic wave input expanded into a Fourier series as:

$e(t) \approx \hat{e}_{0}+\sum_{k=1}^{N} \hat{e}_{2 k-1} \cos \left(\omega_{k} t\right)+\hat{e}_{2 k} \sin \left(\omega_{k} t\right)$

where $\omega_{k}=k \Delta \omega, \Delta \omega=2 \pi / T$ is the frequency step, $T$ is the period of the wave excitation signal, and $N$ is the number of harmonics considered - the cut-off frequency is then $\omega_{c}=2 \pi N / T$.
The instantaneous power, transmitted from the waves to the PTO system, is $-\dot{z}(t) u(t)$. The control problem, over $[0 ; T]$, consists of transmitting as much power as possible from the waves to the PTO system, under the requirement that the dynamical equation (1) is satisfied. Adopting the formalism of a minimisation problem yields:

$$
\begin{aligned}
& \min P(z, u)=\frac{1}{T} \int_{0}^{T} \dot{z}(t) u(t) d t \\
& \text { s.t. } m \ddot{z}=l(z, \dot{z})+n(z, \dot{z})+u(t)+e(t)
\end{aligned}
$$

As in Bacelli and Ringwood (2014), the variables $z$ and $u$ can be approximated using the same basis of harmonic sinusoids used in Eq. (3), as:

$$
\begin{aligned}
& z(t) \approx \hat{z}_{0}+\sum_{k=1}^{N} \hat{z}_{2 k-1} \cos \left(\omega_{k} t\right)+\hat{z}_{2 k} \sin \left(\omega_{k} t\right) \\
& u(t) \approx \hat{u}_{0}+\sum_{k=1}^{N} \hat{u}_{2 k-1} \cos \left(\omega_{k} t\right)+\hat{u}_{2 k} \sin \left(\omega_{k} t\right)
\end{aligned}
$$

Define $\hat{\mathbf{e}}, \hat{\mathbf{z}}$ and $\hat{\mathbf{u}}$ in $\mathbb{R}^{2 N+1}$, the vectors composed of the Fourier coefficients of $e, z$ and $u$ respectively, defined as in Eqs. (3) and (5). Define a block-diagonal matrix $\Omega \in \mathbb{R}^{(2 N+1) \times(2 N+1)}$ :

$\boldsymbol{\Omega}=\left(\begin{array}{cccc}0 & & \cdots & 0 \\ & \boldsymbol{\Omega}_{1} & \cdots & 0 \\ \vdots & \vdots & \ddots & \vdots \\ 0 & 0 & \cdots & \boldsymbol{\Omega}_{N}\end{array}\right)$

where $\forall k \in\{1 \ldots N\}, \boldsymbol{\Omega}_{k}=\left(\begin{array}{cc}0 & \omega_{k} \\ -\omega_{k} & 0\end{array}\right)$

The projection of the velocity $\dot{z}$ onto the Fourier basis is obtained from $\hat{\mathbf{z}}$ as $\hat{\mathbf{z}}=\boldsymbol{\Omega} \hat{\mathbf{z}}$. As shown in Bacelli and Ringwood (2014), the objective function of (4) can then be expressed as

$P(\hat{\mathbf{z}}, \hat{\mathbf{u}})=\frac{1}{2} \hat{\mathbf{z}}^{\mathrm{T}} \boldsymbol{\Omega}^{\mathrm{T}} \hat{\mathbf{u}}$

Furthermore, as explained in Mérigaud and Ringwood (2017) and Mérigaud and Ringwood (0000a), the dynamical equation (1) is also projected onto a Fourier basis, so that $\hat{\mathbf{u}}$ is expressed as a function of the other variables as:

$\hat{\mathbf{u}}(\hat{\mathbf{z}})=\mathbf{M} \hat{\mathbf{z}}-\hat{\mathbf{n}}(\hat{\mathbf{z}})-\hat{\mathbf{e}}$

where:

- $\hat{\mathbf{n}}(\hat{\mathbf{z}})$ represents the $N$ th-order Fourier expansion of $n(z, \dot{z})$, where $z(t)$ and $\hat{\mathbf{z}}$ are related as in Eq. (5);

- The frequency-domain projection of the linear terms $m \ddot{z}-l(z, \dot{z})$ in (1) is given, in matrix form, as M̂̀. 
Typically, when radiation and hydrostatic restoring forces are linearly modelled, $\mathbf{M}$ is a block-diagonal matrix:

$\mathbf{M}=\left(\begin{array}{cccc}s_{h} & & \cdots & 0 \\ & \mathbf{M}_{1} & \cdots & 0 \\ \vdots & \vdots & \ddots & \vdots \\ 0 & 0 & \cdots & \mathbf{M}_{N}\end{array}\right)$

where $\forall k \in\{1 \ldots N\}$,

$\mathbf{M}_{k}=\left(\begin{array}{cc}-\left(m^{\prime}+a_{r}\left(\omega_{k}\right)\right) \omega_{k}^{2}+s_{h} & \omega_{k} b_{r}\left(\omega_{k}\right) \\ -\omega_{k} b_{r}\left(\omega_{k}\right) & -\left(m^{\prime}+a_{r}\left(\omega_{k}\right)\right) \omega_{k}^{2}+s_{h}\end{array}\right)$

where $a_{r}$ and $b_{r}$ are the frequency-dependent radiation added mass and damping, respectively, and $m^{\prime}$ is the device inertia (this time, without taking into account the infinite-frequency radiation added mass).

Combining with (7), the minimisation problem of (4) becomes

$\min \tilde{P}(\hat{\mathbf{z}}):=\frac{1}{2} \hat{\mathbf{z}}^{\mathrm{T}} \boldsymbol{\Omega}^{\mathrm{T}} \hat{\mathbf{u}}(\hat{\mathbf{z}})$

which can be solved using gradient-based optimisation techniques (Mérigaud \& Ringwood, 0000a). The only variables are the components of $\hat{\mathbf{z}}$. The solution $\hat{\mathbf{z}}$ of (10) is the optimal steady-state solution for the problem of (4), within the chosen functional space, i.e. amongst the solutions which can be described as $N$ th-order Fourier series.

The simplifications made to obtain Eq. (10) require that the control input $u$ is the PTO force itself, and can be explicitly written as a function of the other variables through the dynamical equation in (8). In cases where such a simplification is not possible, the dynamic equation must be expressed as a set of equality constraints, as in Bacelli and Ringwood (2014). The reader is referred to Mérigaud and Ringwood (0000a) for more detail.

\subsection{Handling inequality constraints}

Optimal WEC control must allow for handling operational limitations on the device dynamics. Inequality constraints can be expressed in the time domain at a discrete set of $N_{c}$ collocation points $t_{i}, i \in \llbracket 1 ; N_{c} \rrbracket$. Consider a set of linear or non-linear inequality constraints, of the form

$c_{m}\left(z\left(t_{i}\right), \dot{z}\left(t_{i}\right), u\left(t_{i}\right), t_{i}\right) \leq 0$

where $m \in \llbracket 1 ; M_{c} \rrbracket$ and $M_{c}$ is the number of constraints to be satisfied at each time step.

Assuming that $\forall m, c_{m}$ is differentiable, and introducing the nonnegative Lagrange multipliers $\lambda_{m}\left(t_{i}\right)$ associated with the non-linear constraints, the first-order optimality condition (Bazaraa, Sherali, \& Shetty, 2013), for problem (10) with constraints (11), is written as:

$\left\{\begin{array}{l}\nabla \tilde{P}(\hat{\mathbf{z}})+\sum_{m=1}^{M_{c}} \sum_{i=1}^{N_{c}} \lambda_{m}\left(t_{i}\right) \nabla c_{m}\left(t_{i}\right)=0_{\mathbb{R}^{2 N+1}} \\ \forall m, \forall i, \lambda_{m}\left(t_{i}\right) c_{m}\left(t_{i}\right)=0\end{array}\right.$

where the $\nabla$ operator denotes the gradient with respect to the components of $\hat{\mathbf{z}}$, and for brevity $c_{m}\left(t_{i}\right)$ denotes $c_{m}\left(z\left(t_{i}\right), \dot{z}\left(t_{i}\right), u\left(t_{i}\right), t_{i}\right)$.

The constrained problem is solved using an interior-point (IP) algorithm, implemented via the Matlab fmincon function. The gradient (12) of the objective function, as well as its Hessian, can be computed explicitly and efficiently, as detailed in Mérigaud and Ringwood (0000a), resulting in significant computational gain compared to an evaluation of the derivatives through finite differences.

\subsection{Receding-horizon implementation}

The use of FSC calculation techniques presented in this section, in a receding-horizon framework, necessitates specific adaptation due, in particular, to the fact that the excitation force signal, as seen by the RG algorithm within the finite receding horizon is, in general, non-periodic. Receding-horizon calculations using Fourier basis functions are studied in Auger et al. (0000), from which the methodology presented here is adapted. The two main adjustments, required by the FSC method, are as follows (Auger et al., 0000):
- The receding time interval, of duration $T_{w}$, is defined so that the mid-point of the interval (as opposed to its beginning) roughly coincides with the present time, i.e. the time at which the reference trajectory (RT) is updated;

- The section of wave excitation, 'seen' by the RG algorithm within the receding horizon, is windowed by means of a Tukey function (Harris, 1978), in order to make the RG input periodic with no discontinuity at the window ends.

The former adjustment is in contrast to usual predictive control set-up, whereby the present time is at the beginning of the receding window (Richalet, Lavielle, \& Mallet, 2004). The underlying philosophy, governing the choice made here, is to provide an evaluation, as accurate as possible, of the steady-state, optimal WEC trajectory, regardless of where the WEC actually is at the current time, while the task of moving the device towards and along the optimal trajectory is left to the TL. Therefore, when solving the optimisation problem within the receding window, there are no initial or final states to take into account. Furthermore, assuming perfect knowledge of both past and future excitation signals, the optimal, steady-state WEC trajectory, at the present time $t$, depends equally on past and future values of the excitation signal, and therefore there is no reason to 'favour' the future over the past, or vice versa. This approach has clearly proven capable of providing an accurate evaluation of the optimal, steady-state WEC trajectory (Auger et al., 0000).

The RG algorithm updates the RT at regular time instants, say every $\Delta T_{R G}$ seconds. The RG procedure, followed at each update instant (i.e. every $\Delta T_{R G}$ seconds), is illustrated in Fig. 4 , and can be approximately detailed as follows:

(a) Consider a given update instant, where the RG calculations are carried out, and the corresponding receding time interval of length $T_{w}$, comprising both past and future excitation signal values (for the sake of simplicity, in this example it is assumed that both past and future values of $e(t)$ are perfectly known).

(b) The excitation signal, within the receding time interval of width $T_{w}$, is multiplied with a Tukey window.

(c) The resulting signal, $e_{w}(t)$, is projected onto the Fourier basis corresponding to the receding time interval, i.e. using a fundamental frequency $\omega_{1}=2 \pi / T_{w}$, and with a cut-off frequency $\omega_{N}=N \omega_{1}$ which can be adjusted in order to control the degree of smoothness of the optimised trajectory. Using the Fourier projection $\hat{\mathbf{e}}_{w}$ of $e_{w}(t)$, Problem (10) (possibly with constraints (11)) is then solved to yield $\hat{\mathbf{z}}_{w}$ and the corresponding trajectory $z_{w}(t)$ (of period $T_{w}$ ).

(d) Finally, only a small section of $z_{w}(t)$, with length $\Delta T_{R G}$, is appended to the end of the current version of the RT $z_{\text {ref }}(t)$, so as to provide the RT until the next update.

In reality, given the complexity of the non-linear optimisation problem solved by the RG algorithm, it would be unrealistic to consider that the RG calculations can be carried out instantaneously, and thus that the new section of $z_{\text {ref }}$ can become effective at the RG update instant. Instead, some calculation time, $\Delta T_{c}$, is allowed, as illustrated in Fig. 4: the RG calculations starting at time $t_{R G}$ are used to compute $z_{\text {ref }}(t)$ for $t \in\left[t_{R G}+\Delta T_{c} ; t_{R G}+\Delta T_{c}+\Delta T_{R G}\right]$. With such a configuration, the RG calculations can be considered compatible with real-time implementation if the calculation time is lower than $\Delta T_{c}$. Of course, the allowed calculation time $\Delta T_{c}$ must be smaller than the update time $\Delta T_{R G}$. Therefore, a faster RG algorithm allows for more frequent updates of the reference trajectory.

Note that the RT, obtained by concatenating the results of successive RG calculations, may exhibit discontinuities at the switching instant between two successive sections (visible in the bottom graph of Fig. 4). Such discontinuities could result in sudden switching of the control input. Therefore, the updates of $z_{\text {ref }}(t)$ can, in fact, be computed in an overlapping fashion, and a smooth transition ensured between consecutive solutions, as explained below, by means of a transition function. 
(a)

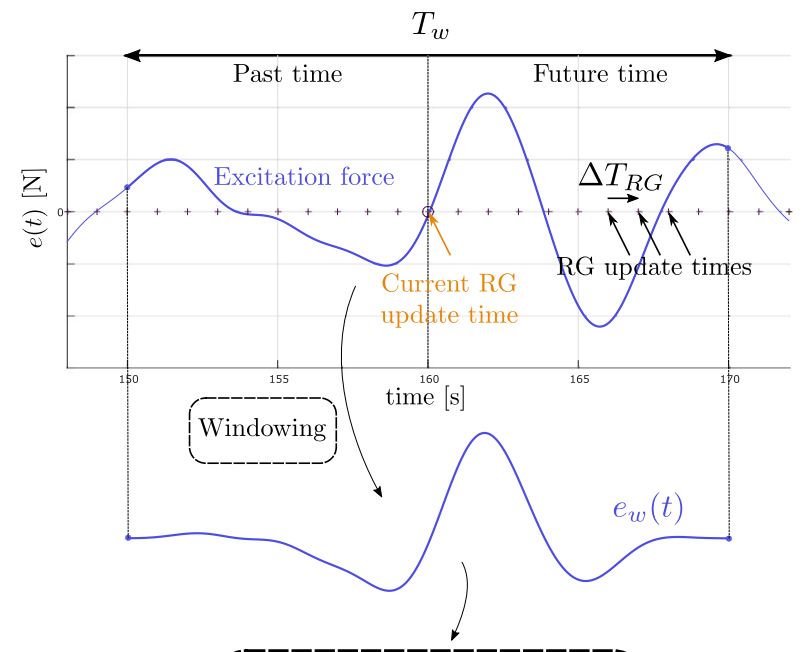

(c)

(d)

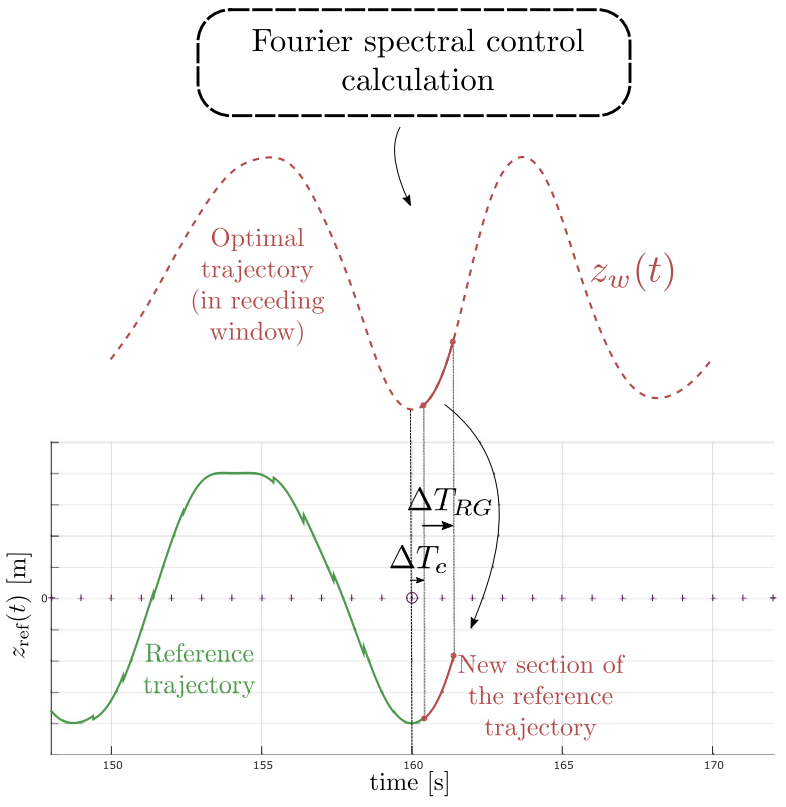

Fig. 4. Receding-horizon implementation of Fourier PS WEC control.

Denote $z_{\text {ref }}(t, k)$ as the RT, calculated at the update instant $t_{R G}=$ $k \Delta T_{R G}$ (bold segment in the bottom graphs of Fig. 4). Instead of covering $t \in\left[t_{R G}+\Delta T_{c} ; t_{R G}+\Delta T_{c}+\Delta T_{R G}\right]$, as in the simplified example of Fig. 4, $z_{\text {ref }}(t, k)$ may in fact cover $t \in\left[t_{R G}+\Delta T_{c} ; t_{R G}+\Delta T_{c}+2 \Delta T_{R G}\right]$. Thus, each time interval $\left[k \Delta T_{R G}+\Delta T_{c} ;(k+1) \Delta T_{R G}+\Delta T_{c}\right]$ is in fact covered by both $z_{\text {ref }}(t, k)$, computed at $k \Delta T_{R G}$, and $z_{\text {ref }}(t, k-1)$, computed at $(k-1) \Delta T_{R G}$.

Define a non-decreasing transition function $\lambda(x)$, defined on $[0 ; 1]$ and taking values in $[0 ; 1]$, such that $\lambda(0)=0$ and $\lambda(1)=1$. For $t \in\left[k \Delta T_{R G}+\Delta T_{c} ;(k+1) \Delta T_{R G}+\Delta T_{c}\right]$, the RT $z_{\text {ref }}(t)$ is computed by weighting $z_{\text {ref }}(t, k-1)$ and $z_{\text {ref }}(t, k)$ as follows:

$$
\begin{aligned}
z_{\mathrm{ref}}(t)= & \lambda\left(\frac{t-k \Delta T_{R G}-\Delta T_{c}}{\Delta T_{R G}}\right) z_{\mathrm{ref}}(t, k) \\
& +\left(1-\lambda\left(\frac{t-k \Delta T_{R G}-\Delta T_{c}}{\Delta T_{R G}}\right)\right) z_{\mathrm{ref}}(t, k-1)
\end{aligned}
$$

The transition function can be linear, or, to also ensure continuity of the 1st-order derivatives, sigmoidal such as $\lambda(x)=\frac{1}{2}+\frac{1}{2} \sin \left(\pi\left(x-\frac{1}{2}\right)\right)$, as illustrated in Fig. 5. Fig. 6 illustrates how, using such a sigmoidal weighting function, a smooth transition is ensured between consecutive RG calculations. In Fig. 6, update instants $k \Delta T_{R G}$ are indicated by coloured markers, which also distinguish the corresponding sections $z_{\text {ref }}(t, k)$. Unlike the RT $z_{\text {ref }}$ in the bottom graph of Fig. 4, the RT $z_{\text {ref }}$ now smoothly transitions across consecutive RG solutions. The

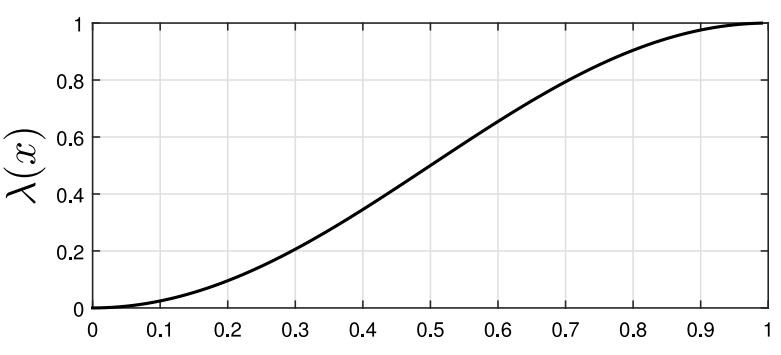

Fig. 5. Smooth transition function: $\lambda(x)=\frac{1}{2}+\frac{1}{2} \sin \left(\pi\left(x-\frac{1}{2}\right)\right)$.

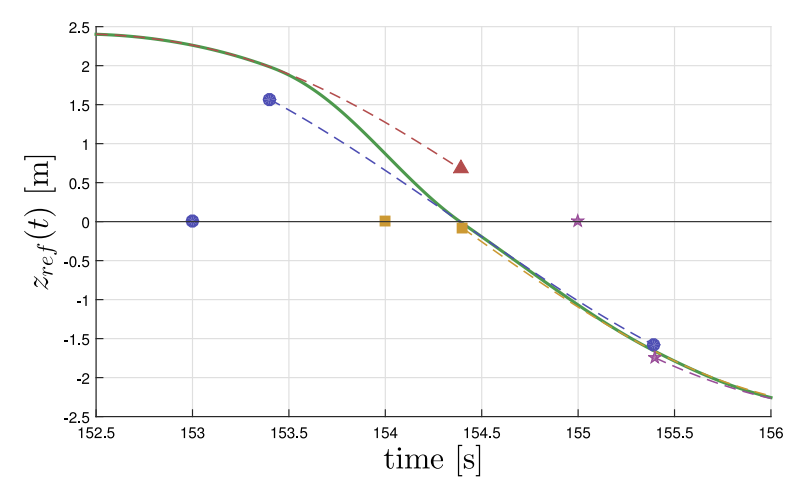

Fig. 6. Sections $z_{\text {ref }}(t, k)$ calculated at update instants $k \Delta T_{R G}$, for four consecutive update instants (dashed lines), and $z_{\text {ref }}(t)$ obtained as in Eq. (13) (solid line). For each $k$, section $z_{\text {ref }}(t, k)$ and calculation instant $k \Delta T_{R G}$ are indicated using the same marker.

sigmoidal transition function of Eq. (13) is used throughout the rest of this paper. However, as will be further exemplified in Section 8, if the difference between successive updates is too sharp, the proposed transition method cannot prevent the occurrence of fast changes in $z_{\text {ref }}$, but avoids discontinuities.

Other possibilities, in terms of RG, have been investigated by the authors:

- Instead of smoothing the RG results ex-post, their continuity can be ensured by the RG algorithm directly. More specifically, an equality constraint is added, requiring that the reference position, velocity and acceleration are continuous at the transition time between the previous and current RG updates. Such a constraint avoids discontinuities between successive updates, and hence obviates the need for any smoothing function. Such a variant is termed Continuous Reference Generation (CRG).

- Alternatively, a choice can be made not to apply any smoothing between successive RG updates, which is termed Discontinuous Reference Generation (DRG).

As will be briefly discussed in Section 8, CRG and DRG both showed significant disadvantages with respect to the RG methodology, presented previously in this section.

Finally, note that the practical issues, related to the discrete updates of the RG calculation, would be strongly mitigated if accurate wave excitation forecasts were available over a longer time horizon: in such a case, RG calculations may be carried out at more distant time intervals; furthermore, discrepancies between successive updates (which result in the observed discontinuities) would also be significantly smaller. However, this possibility is not investigated here, because the general philosophy of this work relies on minimal assumptions with respect to available measurement equipment.

\section{Trajectory tracking loop}

A hierarchical control structure (Fusco \& Ringwood, 2014), articulating state feedback with RG trajectory calculations, provides natural 


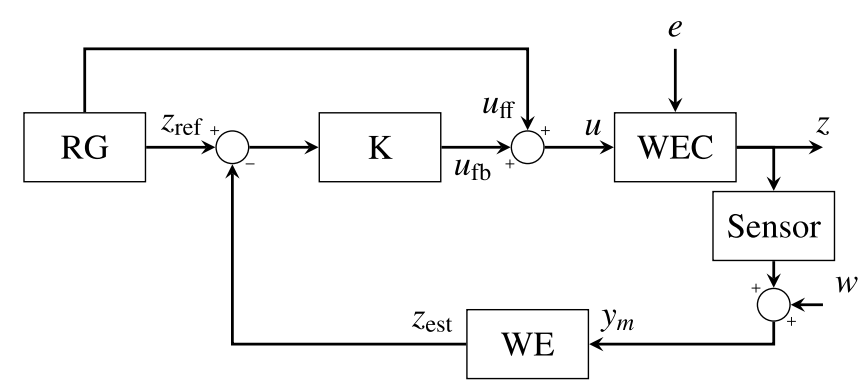

Fig. 7. Structure of the non-linear feed-forward and feedback TL. $z, z_{\text {ref }}, z_{\text {est }}$ : actual, estimated and prescribed trajectory; $w$ : measurement noise; $y_{m}$ : measured WEC output; $e$ : excitation forces; $u_{\mathrm{fb}}, u_{\mathrm{ff}}, u$ : feedback, feed-forward and total control forces.

robustness to some modelling errors, since, as investigated in Mérigaud and Ringwood (2017) and Nielsen et al. (2013), optimal trajectory results are independent of some modelling terms, unlike direct force calculation. In addition, state feedback itself is relatively insensitive to WEC modelling errors since, by making the feedback gains large enough, the steady-state tracking error can be made arbitrarily small (Fusco \& Ringwood, 2014).

However, large gains could also put high demand on the PTO, especially during transient events. In particular, as seen in Section 3, the RT is adjusted at regular time intervals, which can result in relatively abrupt changes in $z_{\text {ref. }}$. Such fast variations are clearly undesirable artefacts, because they are not inherently related to the actual optimal WEC trajectory, but are rather due to its imperfect approximation by the receding-horizon calculations. More importantly, they could result in large excursions of the control input if the feedback gains are large. Such considerations are discussed in Section 8.2.

Furthermore, in practice (as detailed further in Section 5), the system states are not perfectly known, but instead are measured and/or estimated. Large feedback gains also imply a greater sensitivity to measurement noise, of both control input and tracking performance.

In summary, it is desirable to have large control gains in order to ensure accurate tracking of the RT under modelling uncertainties, but some specific issues, arising from the practical controller implementation, also suggest mitigating the value of the control gain. Such trade-offs are illustrated in Section 8.2.

Therefore, it is suggested, in this work, to combine a state feedback term with feed-forward estimation of the necessary steady-state control input, also calculated by the RG algorithm, as illustrated in Fig. 7. For a given trajectory $z_{\text {ref }}$, calculated at the RG level, a feed-forward, nominal control input $u_{\mathrm{ff}}$ is evaluated, using Eq. (1) (or equivalently Eq. (8)). $u_{\mathrm{ff}}$ is applied to the WEC in addition to a feedback term, $u_{\mathrm{fb}}$. The total control force can then be written as:

$$
\begin{aligned}
u(t) & =u_{\mathrm{ff}}(t)+u_{\mathrm{fb}}(t) \\
& =u_{\mathrm{ff}}(t)-k_{1}\left(z_{\text {est }}(t)-z_{\text {ref }}(t)\right)-k_{2}\left(\dot{z}_{\text {est }}(t)-\dot{z}_{\text {ref }}(t)\right)
\end{aligned}
$$

where $k_{1}$ and $k_{2}$ can be computed, for example, using pole assignment. Thus, the role of the state feedback is merely to stabilise the WEC around the RT, and to compensate for possible errors in $u_{\mathrm{ff}}$.

Note that, in Eq. (14), the three terms which originate from the RG level, namely $u_{\mathrm{ff}}(t), z_{\mathrm{ref}}(t)$ and $\dot{z}_{\text {ref }}(t)$, are all calculated using the transition formula of Eq. (13) which, in essence, amounts to smoothing the control law $u(t)$ across successive RG updates.

Other TL possibilities have also been considered by the authors. In particular:

- The TL may solely rely on feedback control, with no feed-forward term. The absence of feed-forward (pro-active) control action, however, necessitates higher requirements on the feedback (reactive) control action, thus necessitating larger control gains to achieve satisfactory tracking performance.
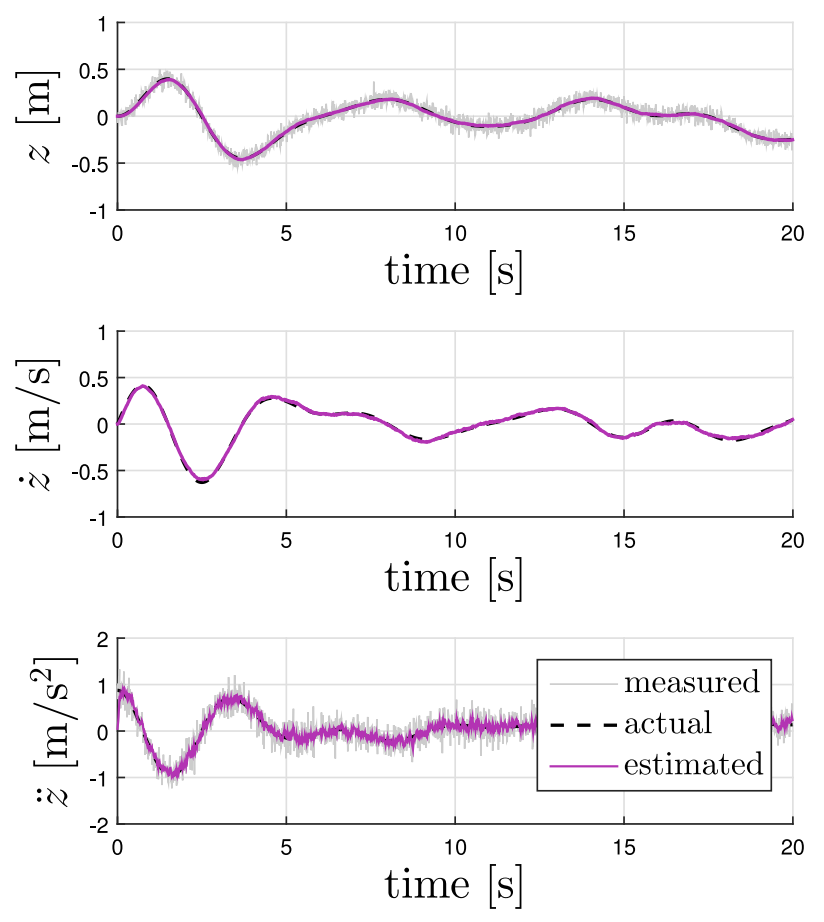

Fig. 8. Kalman filtering for position and velocity estimation.

- Regardless of whether a feed-forward term is used, the state feedback terms on position and velocity errors constitute, in fact, proportional-derivative (PD) control. Position and velocity error terms may be complemented with an integral term on position error, thus resulting in a proportional-integral-derivative (PID) controller. The addition of integral action, with a consequent phase lag, is found to be essential if no feed-forward term is used.

The associated numerical results are not reported in detail in this work, and will only be briefly summarised in Section 8 .

\section{Excitation force estimation}

In this paper, WE is carried out by means of an EKF, solely based on measurements of the WEC acceleration and position. Such an approach requires a state-space representation of the WEC dynamics, in which the excitation force must be included. However, due to the complexity of adding acceleration terms among the system states, WE is carried out in two steps:

- A simple Kalman filter, with no model of the WEC dynamics, is used to estimate $z$ and $z$ from noisy position and acceleration measurements (hereafter denoted $z_{m}$ and $\ddot{z}_{m}$ ), assuming availability of a position sensor and an inertial measurement unit.

- Subsequently, the estimates of $z$ and $\dot{z}$ are used as direct measurements in the EKF, as detailed in Section 5.2.

The approach is similar to that in Abdelkhalik et al. (2016), where velocity is obtained by integrating position, before being used as a direct measurement in an EKF.

\section{1. $K F$ for position and velocity estimation}

The following state-space representation is considered:

$\dot{\mathbf{x}}=\mathbf{A x}$

where

$\mathbf{x}=\left(\begin{array}{c}z \\ \dot{z} \\ \ddot{z}\end{array}\right), \mathbf{A}=\left(\begin{array}{lll}0 & 1 & 0 \\ 0 & 0 & 1 \\ 0 & 0 & 0\end{array}\right)$ 
Such a simple representation is adequate, considering that the KF dynamics will be much faster than those of the excitation force and WEC system.

Eq. (15) is discretised using a zero-order hold ( $\mathrm{ZOH})$ assumption. Taking into account position and acceleration measurements, as well as process and measurement noise, Eq. (15) becomes:

$$
\begin{array}{r}
\mathbf{x}[k+1]=\mathbf{A}^{\prime} \mathbf{x}[k]+\mathbf{w}[k] \\
\mathbf{y}[k]=\mathbf{C x}[k]+\mathbf{v}[k]
\end{array}
$$

where $\mathbf{A}^{\prime}$ is the discrete-time $\mathrm{ZOH}$ version of $\mathbf{A}, \mathbf{w}[k]$ and $\mathbf{v}[k]$ are Gaussian random vectors of appropriate dimensions, and

$\mathbf{C}=\left(\begin{array}{lll}1 & 0 & 0 \\ 0 & 0 & 1\end{array}\right)$

Fig. 8 shows the performance of the proposed KF (in this example, no control is applied to the WEC). As can be seen in Fig. 8, position and velocity estimates are reasonably accurate, in spite of significant measurement noise. The velocity and position resulting from the KF are treated by the EKF as true measurements.

\subsection{EKF for excitation force estimation}

A non-linear state-space model of the WEC is obtained by modelling the radiation memory terms of Eq. (2) in a state-space form of dimension $d_{r}$ (Perez \& Fossen, 2009), where the input to the radiation state-space model is the WEC velocity $\dot{z}$, and the output $f_{r}$ is the radiation memory term:

$$
\begin{array}{r}
\dot{\mathbf{x}}_{r}=\mathbf{A}_{r} \mathbf{x}_{r}+\mathbf{B}_{r} \dot{z} \\
f_{r}=-\mathbf{C}_{r} \mathbf{x}_{r}
\end{array}
$$

where $\mathbf{A}_{r} \in \mathbb{R}^{d_{r} \times d_{r}}, \mathbf{B}_{r}$ and $\mathbf{C}_{r}$ are matrices of appropriate dimensions, and $f_{r}$ is the radiation memory force in Eq. (2). $\mathbf{A}_{r}, \mathbf{B}_{r}$ and $\mathbf{C}_{r}$ may be obtained using different techniques, but here, the recently proposed moment-matching approach (Faedo, Peña-Sanchez, \& Ringwood, 2018) is used. In particular, the property that the quality of the radiation statespace approximation monotonically improves with the chosen order is found appealing.

Furthermore, the excitation force is also included in the state-space representation, assuming some linear internal dynamics, so that:

$$
\begin{aligned}
\dot{\mathbf{x}}_{e} & =\mathbf{A}_{e} \mathbf{x}_{e} \\
e & =\mathbf{C}_{e} \mathbf{x}_{e}
\end{aligned}
$$

where $\mathbf{A}_{e} \in \mathbb{R}^{d_{e} \times d_{e}}$ and $\mathbf{C}_{e}$ are matrices of appropriate dimensions.

Following the ideas of Ling (2015), also investigated in Peña-Sanchez et al. (2018), the excitation forces are represented as a set of harmonic oscillators, but other techniques may be used. However, note that there is no need, within the scope of this work, for a particularly faithful model of the excitation force dynamics, since no WEC modelling errors are considered. In fact, even an over-simplified representation of the excitation force dynamics (e.g. as a constant, or as a system with a constant derivative) was found able to yield acceptable WE - in such a case, the EKF almost entirely relies on the modelled WEC dynamics to provide an excitation force estimate. If modelling errors were to be considered, a more consistent representation of excitation forces would be required, such as in Scruggs, Lattanzio, Taflanidis, and Cassidy (2013). Even so, WE robustness to modelling errors would deserve to be investigated.

Finally, the state-space model, augmented with radiation and excitation force terms, is written as

$$
\begin{array}{r}
\dot{\mathbf{x}}=\mathbf{f}(\mathbf{x})+\mathbf{B} u \\
\mathbf{y}=\mathbf{C x}
\end{array}
$$

with

$$
\mathbf{x}=\left(\begin{array}{c}
z \\
\dot{z} \\
\mathbf{x}_{r} \\
\mathbf{x}_{e}
\end{array}\right), \mathbf{B}=\left(\begin{array}{c}
0 \\
1 / m \\
\mathbf{0}_{d_{r} \times 1} \\
\mathbf{0}_{d_{e} \times 1}
\end{array}\right), \mathbf{C}=\left(\begin{array}{cccc}
1 & 0 & \mathbf{0}_{1 \times d_{r}} & \mathbf{0}_{1 \times d_{e}} \\
0 & 1 & \mathbf{0}_{1 \times d_{r}} & \mathbf{0}_{1 \times d_{e}}
\end{array}\right)
$$

and $\mathbf{f}(\mathbf{x})$ can be decomposed into linear and non-linear terms as

$\mathbf{f}(\mathbf{x})=\mathbf{A x}+\mathbf{n}(\mathbf{x})$

where

$\mathbf{A}=\left(\begin{array}{cccc}0 & 1 & \mathbf{0}_{1 \times d_{r}} & \mathbf{0}_{1 \times d_{e}} \\ -\frac{s}{m} & -\frac{b}{m} & -\frac{\mathbf{C}_{r}}{m} & \frac{\mathbf{C}_{e}}{m} \\ \mathbf{0}_{d_{r} \times 1} & \mathbf{B}_{r} & \mathbf{A}_{r} & \mathbf{0}_{d_{r} \times d_{e}} \\ \mathbf{0}_{d_{e} \times 1} & \mathbf{0}_{d_{e} \times 1} & \mathbf{0}_{d_{e} \times d_{r}} & \mathbf{A}_{e}\end{array}\right)$

and

$\mathbf{n}(\mathbf{x})=\left(\begin{array}{c}0 \\ \frac{n\left(\mathbf{x}_{1}, \mathbf{x}_{2}\right)}{m} \\ \mathbf{0}_{d_{r} \times 1} \\ \mathbf{0}_{d_{e} \times 1}\end{array}\right)$

where $n$ is defined as in Eq. (1). The terms $-\frac{s}{m}$ and $-\frac{k}{m}$ represent any linear stiffness and damping terms respectively. For example, if the hydrostatic restoring force is linearly modelled, $s$ corresponds to $s_{h}$, the hydrostatic stiffness.

The continuous-time state-space model of Eq. (21) is discretised, assuming a $\mathrm{ZOH}$ and the Euler integration rule. Note that the 'measurements' $\mathbf{y}[k]$ for position and velocity are, in fact, the estimates from the KF presented in Section 5.1.

\section{Excitation force forecasting}

A cost-effective approach to short-term wave forecasting consists of treating the wave signal as a time series (Fusco \& Ringwood, 2010), using past measurements at the point of interest (in this case, the WEC location) to predict the incoming signal. This section, adapted from (Mérigaud \& Ringwood, 0000b), shows how the Gaussian description of ocean waves can be used to provide a simple, statistically-optimal predictor. While, in Mérigaud and Ringwood (0000b), wave elevation observations are used to compute wave elevation predictions, in this work, excitation force observations (e.g. obtained as in Section 5.2) are used to compute excitation force predictions. In other words, the variable of interest is the excitation force (instead of wave elevation).

Consider the wave elevation, $\eta$, modelled as a stationary, ergodic Gaussian process (Ochi, 2005), with spectral density function (SDF) $S_{\eta \eta}(\omega)$. Assuming linear hydrodynamic interactions, the excitation force $e$ is also a stationary, ergodic Gaussian process. Defining $H_{\eta e}(\omega)$ the transfer function relating $\eta$ to $e$, the excitation force process has a SDF $S_{e e}(\omega)=\left|H_{\eta e}(\omega)\right|^{2} S_{\eta \eta}(\omega)$ (Papoulis \& Pillai, 2002). The process $e$ is entirely characterised by its mean $(\bar{e}=0)$ and its auto-covariance function (ACVF) $R_{e e}(\tau)$ for $\tau \in \mathbb{R}$ :

$R_{e e}(\tau)=\mathbb{E}[e(t) e(t+\tau)]$

$R_{e e}(\tau)$ can be computed from $S_{e e}(\omega)$ by means of a Fourier transform, by virtue of the Wiener-Khintchine theorem (Ochi, 2005).

Following the definition of a Gaussian random process, any finite, discrete ensemble of wave excitation values, taken at various points in time, forms a multivariate, Gaussian random vector. Considering that the wave elevation is sampled, say, every second, define $\mathbf{p}$ as the vector of the last $N_{p}$ recorded values (indexed by $m \in \llbracket 1 ; N_{p} \rrbracket$ ), and $\mathbf{q}$ the (unknown) vector of the next $N_{q}$ excitation force values (indexed by $\left.n \in \llbracket 1 ; N_{q} \rrbracket\right)$.

Altogether, the $N_{p}+N_{q}$ points form a multivariate Gaussian random vector, $\mathbf{v} \in \mathbb{R}^{N_{p}+N_{q}}$. Its mean is $0_{\mathbb{R}^{N_{p}+N_{q}}}$ and its variance-covariance matrix, denoted $\Sigma_{\mathbf{v v}}$, can be entirely derived from the correlation values between any pair of points in time, or the wave spectrum, i.e. $\boldsymbol{\Sigma}_{\mathbf{v v} i, j}=$ $R_{e e}\left(t_{j}-t_{i}\right)$.

$\Sigma_{\mathrm{vv}}$ can be written as:

$\Sigma_{\mathrm{vv}}=\left(\begin{array}{cc}\Sigma_{\mathrm{qq}} & \boldsymbol{\Sigma}_{\mathrm{qp}} \\ \boldsymbol{\Sigma}_{\mathrm{pq}} & \boldsymbol{\Sigma}_{\mathrm{pp}}\end{array}\right)$ 


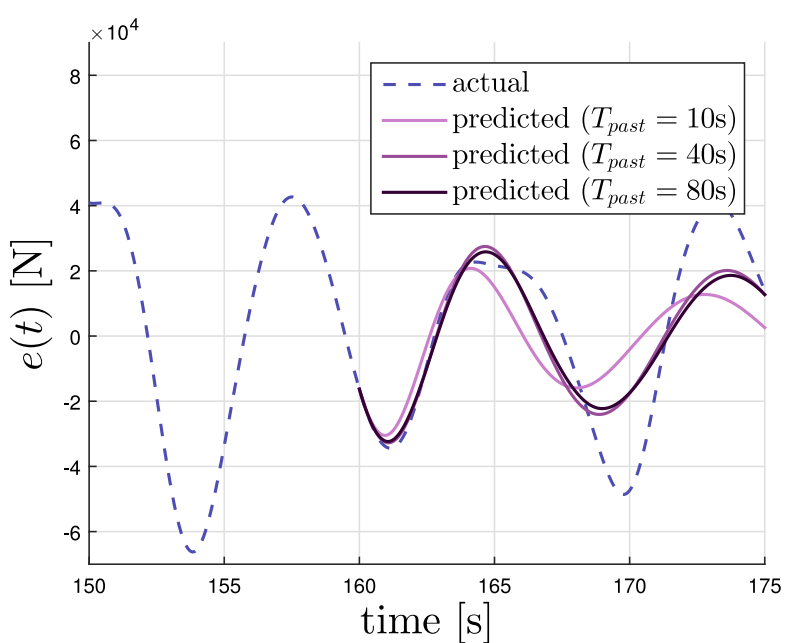

Fig. 9. Actual vs. predicted excitation force, using past measurements over 10, 40 and $80 \mathrm{~s}$, respectively JONSWAP spectrum $\left(H_{m_{0}}=1 \mathrm{~m}, T_{p}=9 \mathrm{~s}\right)$.

where $\boldsymbol{\Sigma}_{\mathbf{q p}}=\boldsymbol{\Sigma}_{\mathbf{p q}}^{\mathrm{T}}$. Using $\mu_{\mathbf{p}}=\mu_{\mathbf{q}}=0$, the conditional distribution of $\mathbf{q} \mid \mathbf{p}$ is multivariate Gaussian (see for example Eaton, 2007) with mean:

$\mu_{\mathbf{q} \mid \mathbf{p}}=\Sigma_{\mathbf{q p}} \Sigma_{\mathbf{p p}}^{-1} \mathbf{p}$

and variance:

$\Sigma_{\mathrm{q} \mid \mathrm{p}}=\Sigma_{\mathrm{qq}}-\Sigma_{\mathrm{qp}} \Sigma_{\mathrm{pp}}^{-1} \Sigma_{\mathrm{pq}}$

The best predictor of $\mathbf{q}$, in a least mean-square sense, is given as

$\tilde{\mathbf{q}}=\mu_{\mathbf{q} \mid \mathbf{p}}=\boldsymbol{\Sigma}_{\mathbf{q p}} \boldsymbol{\Sigma}_{\mathbf{p p}}^{-1} \mathbf{p}$

The prediction matrix, $\mathbf{Q}:=\boldsymbol{\Sigma}_{\mathbf{q p}} \boldsymbol{\Sigma}_{\mathbf{p p}}^{-1}$, which maps $N_{p}$ measured values to $N_{q}$ predicted values, needs only be computed once for a given sea condition, i.e. as the wave spectrum evolves significantly, for example every $30 \mathrm{~min}$. Therefore, the only operation to be carried out in real time is the matrix multiplication $\tilde{\mathbf{q}}=\mathbf{Q p}$. The mean-square prediction error $\epsilon^{2}(h)$, for each prediction horizon $h$, is given by the diagonal terms of $\boldsymbol{\Sigma}_{\mathbf{q} \mid \mathbf{p}}$. For a given order $N_{p}$, any other forecasting method is sub-optimal with respect to the law derived in Eq. (30), to evaluate the $N_{q}$ predicted points.

Finally, it has to be noted that, in practice, the observed excitation values come from the estimator of Section 5.2, and therefore the values observed in vector $\mathbf{p}$ are noisy. Assuming that estimation errors are white noise, they can be readily taken into account, by adding the appropriate variance level to the diagonal terms of $\boldsymbol{\Sigma}_{\mathbf{p p}}$.

Figs. 9 and 10 give examples of WF performed by the SBP when, respectively, 10, 40 and $80 \mathrm{~s}$ of past, measured excitation force values (vector $\mathbf{p}$ ) are used for WF over the next $15 \mathrm{~s}$ of excitation force signal (vector q). The wave condition is a JONSWAP spectrum (Hasselmann, Barnett, Bouws, Carlson, Cartwright, Enke, et al., 1973), with $H_{m_{0}}=1$ $\mathrm{m}$ and $T_{p}=9 \mathrm{~s}$, and the WEC is the spherical heaving point-absorber (HPA) which will be detailed in Section 7. Fig. 10 shows the goodness of fit (GoF) of the prediction corresponding to the three measurement configurations, computed as

$G(h)=1-\sqrt{\frac{\epsilon^{2}(h)}{\mathbb{E}\left[e^{2}\right]}}$

Note that the results of Fig. 10 do not depend on the specific instance of the prediction, but represent the average performance of the predictor. As can be seen in Figs. 9 and 10, significant improvement is achieved by considering $40 \mathrm{~s}$ of past values instead of 10 , while it seems that little accuracy benefits can be expected by using a longer history (e.g. 80 s) of the past values to perform WF. The example of Figs. 9 and

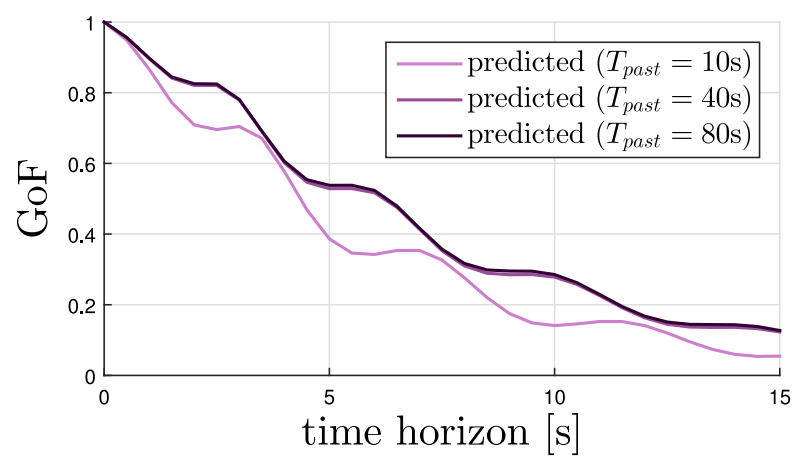

Fig. 10. Average goodness of fit of the excitation force prediction, using past measurements over 10, 40 and $80 \mathrm{~s}$, respectively JONSWAP spectrum $\left(H_{m_{0}}=1 \mathrm{~m}, T_{p}=9 \mathrm{~s}\right)$.

10 does not consider the case where the observed excitation force values are noisy.

It should be pointed out that, like the SBP, the AR-based WF technique (Brekken, 2011; Fusco \& Ringwood, 2010; Peña-Sanchez et al., 2018; Tona et al., 2015) is also consistent with the assumption of Gaussian, linear waves, but differs in two respects from the SBP:

- With the AR model, the WF structure is linear for the one-stepahead predictor, and WF at further time steps are carried out iteratively. In contrast, SBP directly yields one linear predictor for each time horizon.

- AR coefficients are generally identified using past data, while SBP directly identifies the prediction coefficients from the spectrum, without requiring any data-based identification procedure. In theory, if properly identified, the AR coefficients should coincide with the coefficients of the SBP, for the 1-step-ahead predictor.

\section{WEC model and simulation framework}

\subsection{WEC model and constraints}

For the practical application of the control architecture and techniques proposed in this paper, the WEC model considered is the same $5 \mathrm{~m}$ diameter, spherical HPA as in Mérigaud and Ringwood (2017), illustrated in Fig. 11. The vertical position $z$ of the WEC gravity center, $G$, with respect to the still water level, is assumed to be the only degree of freedom. The sphere density is half of the water density so that, at rest, $G$ is on the plane $z=0$. The radiation and excitation forces are represented linearly, and the frequency-domain coefficients for radiation and excitation forces are computed using the hydrodynamic software NEMOH. ${ }^{1}$ Furthermore, in the numerical simulation, radiation forces are computed by means of a convolution product, as in Eq. (2) (where the radiation kernel $k_{r}(\tau)$ is also obtained from NEMOH).

The hydrostatic restoring force is non-linearly modelled, taking into account the actual position of the device with respect to the plane $z=0$, as in Nielsen et al. (2013):

$f_{h s}(z)= \begin{cases}\pi \rho g\left(\frac{1}{3} z^{3}-R^{2} z\right) & z \in[-R ; R] \\ -\frac{2}{3} \pi \rho g R^{3} & z \geq R \\ \frac{2}{3} \pi \rho g R^{3} & z \leq-R\end{cases}$

where $R=2.5 \mathrm{~m}$ is the sphere diameter and $g$ the acceleration due to gravity. Furthermore, a quadratic viscous drag term is added to the model, of the form $f_{v}(\dot{z})=-b_{v} \dot{z}|\dot{z}|$. Thus, the non-linear terms of Eq. (1) are expressed as:

$n(z, \dot{z})=f_{h s}(z)+f_{v}(\dot{z})$

1 https://lheea.ec-nantes.fr/doku.php/emo/nemoh/start. 


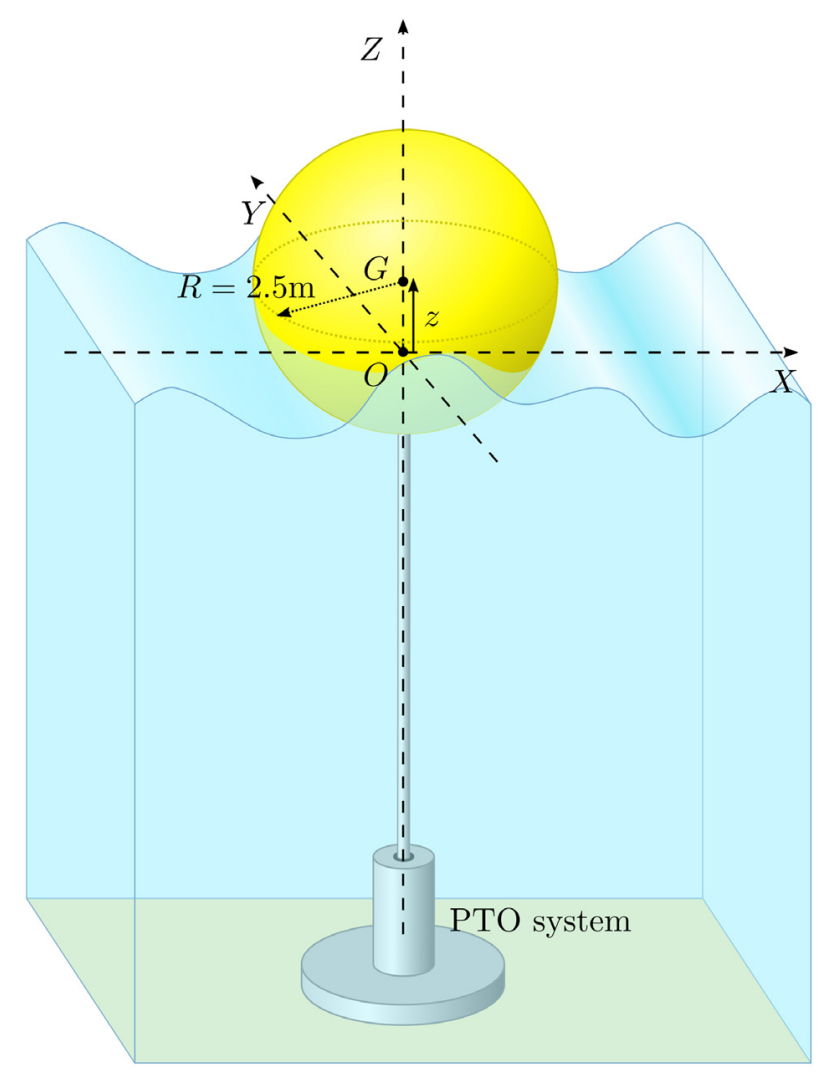

Fig. 11. A spherical heaving point absorber with centre of gravity $G$, radius $R=2.5 \mathrm{~m}$, and density equal to $50 \%$ of that of sea water.

Thus, as in Mérigaud and Ringwood (2017), a mix of static and velocity-dependent forces is considered. Two constraint configurations are explored:

- Variant 1: with position and velocity limitations $\left(|z|_{\max }=2.4 \mathrm{~m}\right.$, $|\dot{z}|_{\max }=2.4 \mathrm{~m} / \mathrm{s}$ ), chosen to prevent the device from being either fully submersed or fully "dry";

- Variant 2: Like Variant 1, with the additional constraint of passivity, i.e. unidirectional power flow.

Variant 2 is particularly demanding, since it involves a highly non-linear constraint, and because a relatively large number of collocation points for the satisfaction of the inequality constraint (see Section 3.2), must be specified.

\subsection{Control performance assessment}

In order to assess the impact of the various parameters and techniques considered in this work, several receding-horizon control variants are examined:

C1 Assuming perfect knowledge of present and future excitation force;

C2 Assuming perfect knowledge of present excitation force but (imperfect) WF, carried out as in Section 6;

C3 Assuming (imperfect) WE carried out as in Section 5, and (imperfect) WF carried out as in Section 6. Different measurement noise levels are examined. As in Fig. 3, C3 is illustrated in Fig. 12, this time with the detail of the chosen techniques presented in Sections 3 through 6.

Two other points of comparison are provided:

- When possible, the optimal (steady-state) trajectory and power output are calculated off-line, over the whole simulation duration, using the FSC technique detailed in Section 3. If the simulation duration is $T_{\text {sim }}$, the generated excitation force signal is periodic with period $T_{\text {sim }}$, and the techniques of Section 3 can be applied, with a fundamental frequency, for the Fourier basis, defined as $\omega_{1}=2 \pi / T_{\text {sim }}$. The corresponding optimisation problem is, of course, significantly more demanding than that solved in any given receding window. Without the passivity constraint, the offline calculation can be carried out in a few seconds only, for a simulation duration $T_{\text {sim }}=200 \mathrm{~s}$. With the passivity constraint, however, off-line calculations cannot always be carried out within a reasonable amount of time. Therefore, the simulation time for the passive case is restricted to $T_{\text {sim }}=180 \mathrm{~s}$, which reduces the number of cases where the overall optimal results cannot be provided.

- Finally, the power obtained using an optimally-tuned constant, passive linear damper is also calculated for each sea state considered.

\subsection{Numerical simulation and control set-up}

Given the large number of parameters to consider in the proposed control architecture, it was not possible to carry out simulations across a large range of sea states. Only five JONSWAP spectra (Hasselmann et al., 1973) are used, with the same $H_{m_{0}}=1 \mathrm{~m}$ and $T_{p}=6,7,8,9$ and $10 \mathrm{~s}$, representative of a location such as Galway Bay (Mérigaud \& Ringwood, 0000c) (given the relatively small dimensions of the device considered, such a location, with modest sea states, would be adequate).

Simulations are carried out using a 2nd-order Runge-Kutta integration method. The time-scales associated with the simulation and the different blocks of the control structure are detailed as follows:

- The simulation time-step is $\Delta T_{R K}=0.01 \mathrm{~s}$, which allows for accurate results given the relatively slow dynamics of typical ocean waves.

- For simplicity, the same time-step is used for the KF and EKF estimators introduced in Section 5: $\Delta T_{R K}=\Delta T_{W E}$ (both KF and EKF calculations are carried out significantly faster than $0.01 \mathrm{~s}$, so that the choice $\Delta T_{W E}=0.01 \mathrm{~s}$ is compatible with real-time implementation).

- Again for simplicity, the control force (comprising a feed-forward and a feedback term, as explained in Section 4) is applied with a time step $\Delta T_{u}=\Delta T_{W E}=\Delta T_{R K}$. Zero-order-hold is assumed over the duration $\Delta T_{U}$.

- The forecasting method, introduced in Section 6, uses a timestep of $\Delta T_{W F}=0.5 \mathrm{~s}$, and takes into account $100 \mathrm{~s}$ of past values to determine the WF: $\Delta T_{W F}$ corresponds to a Nyquist frequency of $1 \mathrm{~Hz}$, which allows the frequency content of ocean waves to be captured, while taking $100 \mathrm{~s}$ of past values is more than sufficient to obtain the best possible forecasts (Mérigaud \& Ringwood, 0000b). Thus, the vector of observed values $\mathbf{p}$ contains samples every $0.5 \mathrm{~s}$ from $t-100$ to $t$, i.e. $1+100 / 0.5=201$ samples (where $t$ is the present time, at which WF is carried out). The size of the predicted vector depends, of course, on the time horizon considered - for example, if the next $10 \mathrm{~s}$ are being forecast, the predicted vector $\mathbf{q}$ contains 20 values. The prediction matrix $\mathbf{Q}$ is then of size $20 \times 201$. The prediction, carried out before each RG update by means of a simple matrix multiplication, represents a negligible computational burden with respect to the RG calculation itself.

- The time step $\Delta T_{R G}$, at which the control calculations are carried out (see Section 3), can be adjusted depending on the case considered, in particular depending on the complexity of the RG control calculations, and on the quality of the excitation forecasts. Ideally, small values are preferable, because they allow for new excitation forecasts to be taken into account as soon as they become available, and because they imply that the discrepancies between two successive RG updates are smaller, hence mitigating 


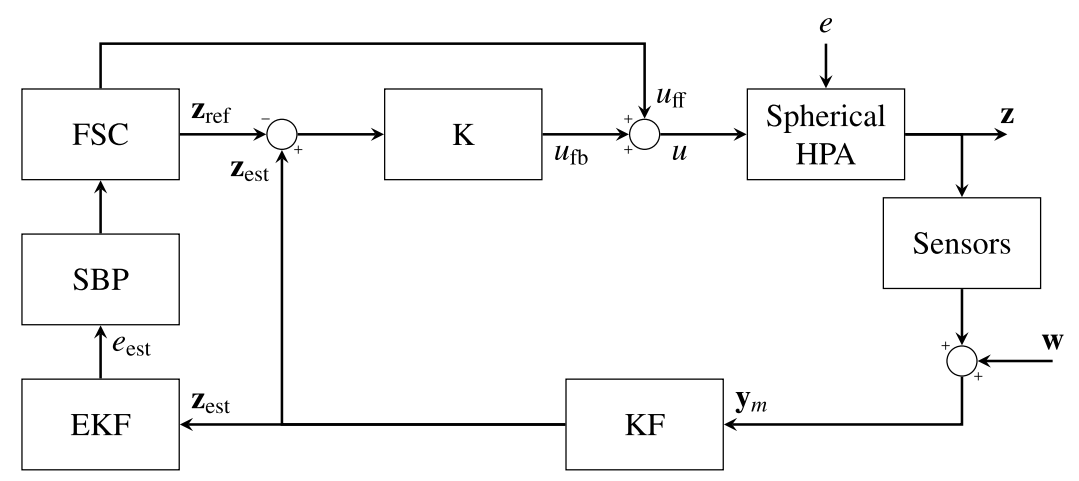

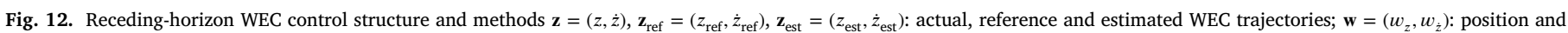
acceleration measurement noise; $\mathbf{y}_{m}=\left(z_{m}, \ddot{z}_{m}\right)$ : measured WEC outputs; $e, e_{\mathrm{est}}$ : actual and estimated excitation forces; $u_{\mathrm{fb}}, u_{\mathrm{ff}}, u$ : feedback, feed-forward and total control input.

discontinuities in the reference trajectories. However, $\Delta T_{R G}$ must also be compatible with real time RG calculations. Throughout the rest of this paper, $\Delta T_{R G}$ is set to $0.25 \mathrm{~s}$, which is a satisfactory compromise. In all cases, the allowed calculation time $\Delta T_{c}$ (see Section 3) is set to $0.2 \mathrm{~s}$.

The settings for the receding-horizon RG calculations have been manually tuned, and are detailed as follows: The receding window length, $T_{w}$, and the cut-off frequency of the RG calculation, are important drivers for controller performance. Overall, it is found that, with a bi-directional power flow, a window length $T_{w}=3 T_{p}$, where $T_{p}$ is the peak wave period, and a cut-off frequency for the Fourier basis, $f_{c}=0.5 \mathrm{~Hz}$, are a reasonable compromise, between quality of the generated trajectory and computational speed. In the passive case (also significantly more computationally demanding), $T_{w}=2 T_{p}$ seems a sufficient window length, but a significantly higher cut-off frequency is required in order to obtain acceptable results; the effect of the cut-off frequency will be investigated further in Section 8 .

Finally, note that the receding-horizon solution $\hat{\mathbf{z}}_{w, k}$, obtained by the RG algorithm, at update time $k \Delta T_{R G}$, is used as a starting guess for the solution of the RG problem at update time $(k+1) \Delta T_{R G}$, after appropriate time-shifting by $\Delta T_{R G}$. Such a starting point generally reduces the number of iterations for the interior point algorithm to converge, and thus results in considerable computational savings.

All simulations and control calculations are carried out in a Matlab ${ }^{2}$ environment, using a computer equipped with a $3.50 \mathrm{GHz}$, 8-core Intel ${ }^{\circledR}$ processor.

\section{Numerical results}

\subsection{Measurements and estimation}

Figs. 13a and 13b examine the performance of the KF and EKF for state estimation and WE, under two, arbitrarily chosen noise level scenarios: respectively small $\left(\sigma_{z}=0.025, \sigma_{\ddot{z}}=0.1\right)$ and larger $\left(\sigma_{z}=\right.$ $\left.0.05, \sigma_{\ddot{z}}=0.2\right)$. Furthermore, two types of dynamics are considered: uncontrolled (on the left hand-side of each figure), and controlled (right hand-side).

For the uncontrolled case, $u(t)$ is simply set to zero, so that the WEC moves freely in the waves. For the controlled condition, the optimal trajectory $z_{\text {ref }}=z_{\text {opt }}$ is computed off-line over a wave signal of period $T_{\text {sim }}=200 \mathrm{~s}$ (assuming perfect knowledge of $e(t)$ ), allowing for bidirectional power flow. Then, in a simulation, the WEC is stirred along $z_{\text {opt }}$ using the TL of Section 4. Thus, the impact of measurement noise can be illustrated, in controlled conditions, while avoiding any complicated interaction with RG calculations.

Reactive control implies larger WEC motion, and therefore the impact of measurement noise can appear to be less significant under

\footnotetext{
2 https://uk.mathworks.com/.
}

controlled conditions (if the measurement noise magnitude remains the same). This is particularly visible by contrasting the top-left and top-right graphs in Fig. 13b. Unsurprisingly, additional measurement noise results in more noisy excitation force estimates, as can be seen by comparing the bottom graphs of Figs. 13a and 13b. The impact of measurement noise and WE errors, on the complete control loop of Fig. 12, will be further illustrated in the numerical results of Sections 8.2 and 8.3 .

\subsection{Reference trajectory and tracking}

Figs. 14a and 14b illustrate the importance of appropriate TL settings, and their interaction with the RG control calculations. Three trajectories are represented on the upper graphs of both Figs. 14a and 14b: the RT, generated as explained in Section 3; the actual trajectory, followed by the WEC as a result of the non-linear feed-forward and linear state feedback described in Section 4, and the optimal WEC trajectory, computed off-line using the totality of the $200 \mathrm{~s}$ of simulation. The middle graphs show the optimal control force, the feed-forward control force $u_{\mathrm{ff}}$ computed as a result of the RG optimisation, and the actual control force, resulting from both $u_{\mathrm{ff}}$ and $u_{\mathrm{fb}}$. The bottom graphs show the actual excitation force, and that estimated by the EKF (Section 5.2). The time span indicated (from $300 \mathrm{~s}$ to $350 \mathrm{~s}$ ) is because the time-domain simulation is, in fact, carried out in two successive 200-s periods of the generated excitation signal, thus allowing for a steady-state analysis between $t=200 \mathrm{~s}$ and $t=400 \mathrm{~s}$. The measurement noise is the smaller one $\left(\sigma_{z}=0.025, \sigma_{\ddot{z}}=0.1\right)$, presented in Section 8.1.

In Fig. 14a, the control gains $k_{1}$ and $k_{2}$ (see Eq. (14)) are set so that the closed-loop system (with a 2nd-order approximation of the WEC model) has both poles, $p_{1}$ and $p_{2}$, equal to -4 . Such a choice results in relatively large values for the control gains, especially $k_{1}\left(k_{1} \approx 600000\right)$, which makes the controller over-react to the short-term dynamics of the RT. In the example of Fig. 14a, this is particularly visible between $t=310$ and $315 \mathrm{~s}$, but in other parts of the signal the good TL performance is achieved at the price of significant excursions in the control signal. The rapid WEC oscillations have a detrimental impact on WE (bottom graph), which, in turn, affects RG calculations, as seen from the rapid oscillations exhibited by the RT in the top graph. The resulting control force oscillations have large amplitudes and are clearly unacceptable.

In Fig. 14b, the control gains are adjusted so that $p_{1}=p_{2}=-2$. Although $z_{\text {ref }}$ is, at times, more loosely followed, thus possibly resulting in small excursions outside the constraints (see for example $t=347$ ), the control force dynamics and WE are now acceptable. It can also be seen that the feed-forward control input $\left(u_{\mathrm{ff}}\right)$, represented as a dotted line in the middle graph, is the dominant term in the total control force, with feedback playing a minor role.

For the remainder of the numerical results presented in this section, the choice $p_{1}=p_{2}=-2$ is maintained: even though better trade-offs could probably be found, such a choice yields satisfactory results over the range of conditions considered, and simplifies the analysis. 

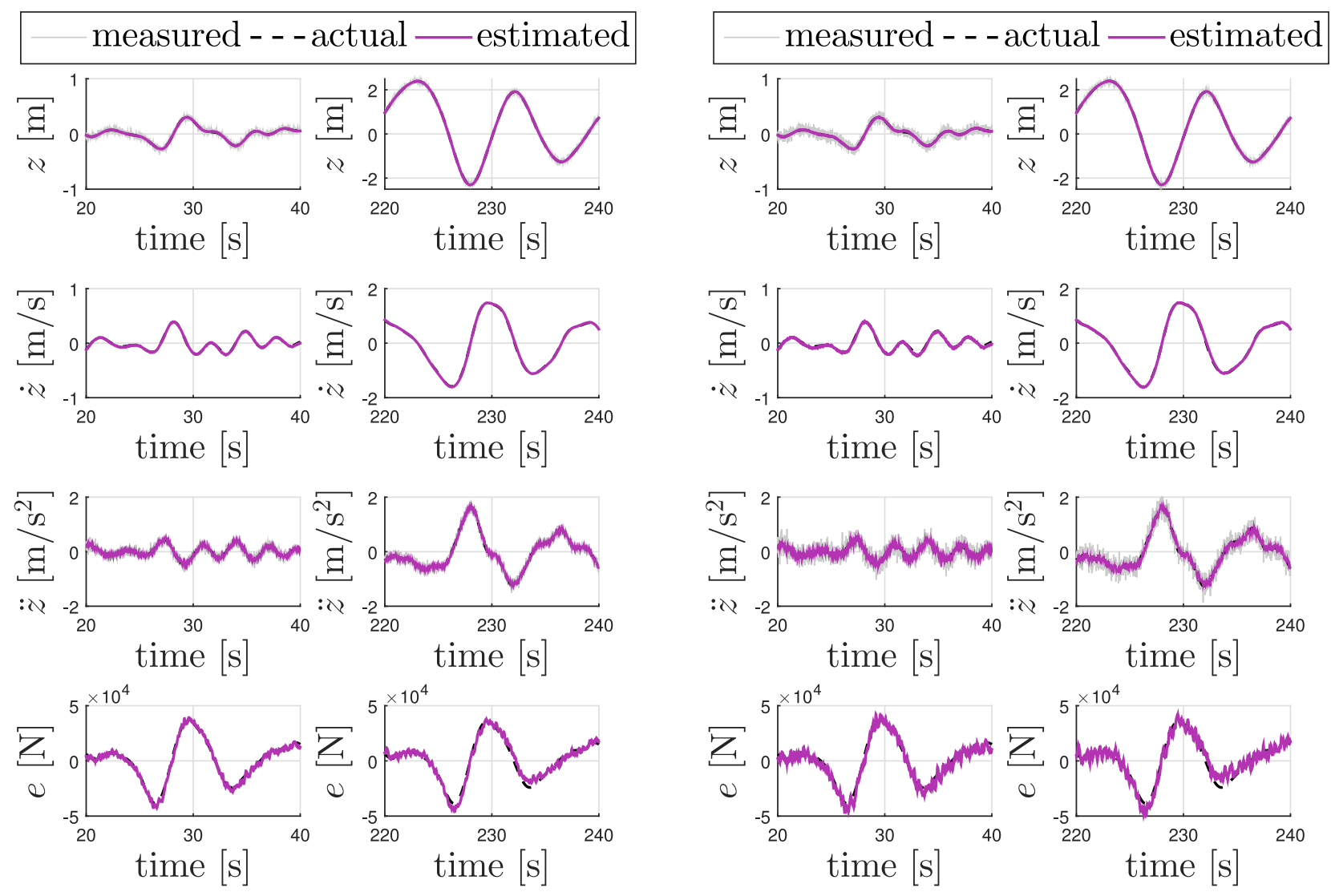

(a) Small measurement noise $\left(\sigma_{z}=0.025, \sigma_{\ddot{z}}=0.1\right)$. Uncontrolled (left) $v s$ controlled (right)

(b) Larger measurement noise $\left(\sigma_{z}=0.05, \sigma_{\ddot{z}}=0.2\right)$. Uncontrolled (left) $v s$ controlled (right)

Fig. 13. Actual and estimated values for position, velocity, acceleration and excitation force (along with position and acceleration measurements).

As mentioned in the end of Section 4, other TL options, different from the proposed feed-forward and state feedback of Eq. (14), may be considered. Adding an integral term in the reactive part of the control action was found to be a valid option - even though it did not significantly change results with respect to the TL controller retained. In contrast, removing the feed-forward part of the controller, $u_{\mathrm{ff}}$, was found detrimental. Without $u_{\mathrm{ff}}$, large feedback control gains were necessary for accurate trajectory tracking, both in a PD and PID configuration. As a consequence, however, the resulting control signal $u$ showed unacceptably large excursions.

Finally, note that the high-frequency oscillations observed in Fig. $14 \mathrm{a}$, and to a lesser extent in Fig. 14b, result from the interaction between the TL and the RG. Changing the RG update rate, or managing transitions between updates in a manner other than the one proposed in Section 3.3, would yield different results. In particular, the two alternative possibilities mentioned in the end of Section 3.3, namely CRG and DRG, have been briefly investigated:

- With CRG, the reference trajectory is indeed smooth across successive updates; however the equality constraint, expressing the continuity of the reference trajectory, makes the optimisation significantly more difficult, and thus incompatible with real-time calculations. More importantly, the reference trajectory calculated with CRG is further from the actual optimal trajectory, than that calculated without the continuity constraint. This can be interpreted as follows: with CRG, the errors generated, in computing the reference trajectory at update time $t=k \Delta T_{R G}$, have an impact on the calculations made at the next update, at $t=(k+1) \Delta T_{R G}$. In contrast, if no continuity constraint is added (as is the case for the results presented in this paper), the RG solution at $t=(k+1) \Delta T_{R G}$ is completely decoupled from that at the previous update, and thus is not 'contaminated' by previous errors.

- With DRG, no smoothing or continuity constraints are applied to the successive RG solutions. The resulting performance, in terms of power output, is similar to the performance of the controller with smoothing, but the corresponding control signal $u(t)$, computed as in Eq. (14), is non-smooth, which is clearly undesirable.

\subsection{Controller performance}

With appropriate settings for the TL, the controller performance, in terms of power absorption, is assessed over the range of sea states considered, both with and without the possibility of bi-directional power flow between the WEC and PTO system.

\section{With bi-directional power flow}

Fig. 15 shows the average steady-state absorbed power, for the different variants detailed in Section 7.2, assuming that a bi-directional power flow is allowed. For Variant $\mathrm{C} 3$, two measurement noise level scenarios are considered: small $\left(\sigma_{z}=0.025, \sigma_{\ddot{z}}=0.1\right)$ and larger $\left(\sigma_{z}=\right.$ $0.05, \sigma_{\ddot{z}}=0.2$ ). The noise level, which has to be taken into account in the SBP (see Section 6), should be adapted to the level of noise observed in the WE (bottom graphs in Figs. 13a and 13b). Better still, similarly to spectrum-based prediction, information on the spectrum could be used to filter noise out of the estimated excitation signal, hence possibly improving the quality of the input $e_{w}$ of the RG calculations. However, such developments are beyond the scope of the current work, 

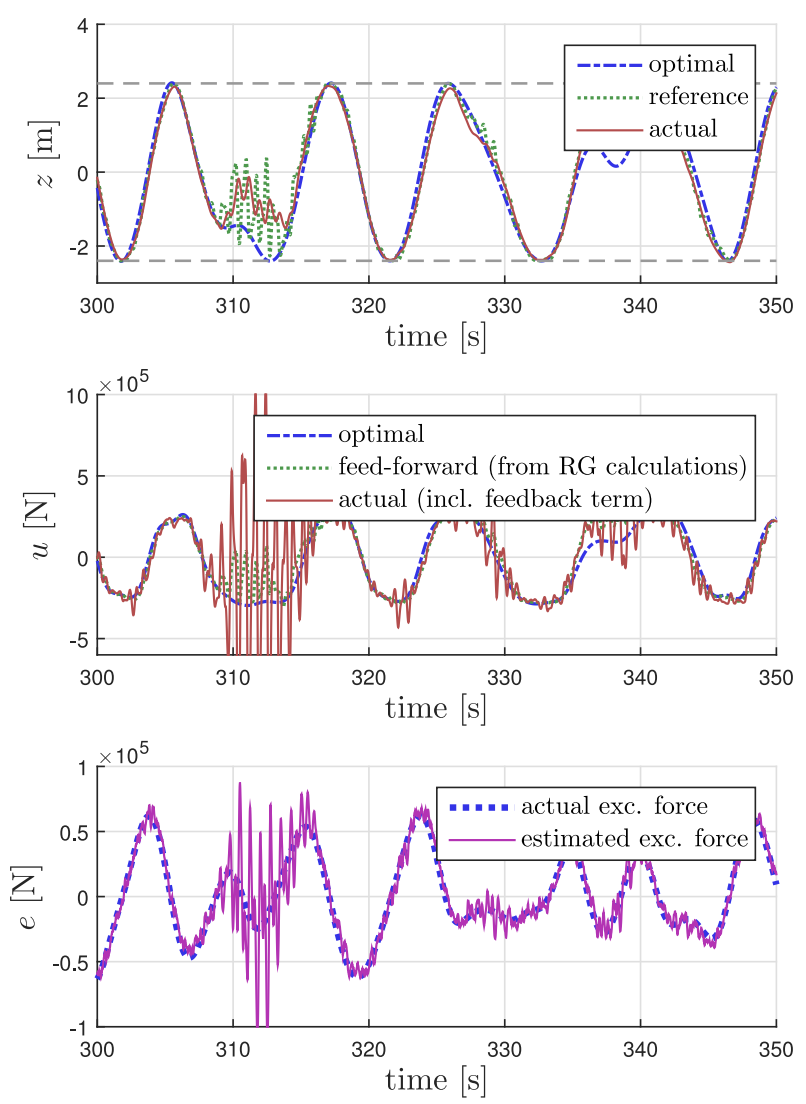

(a) Poles $p_{1}=p_{2}=-4$
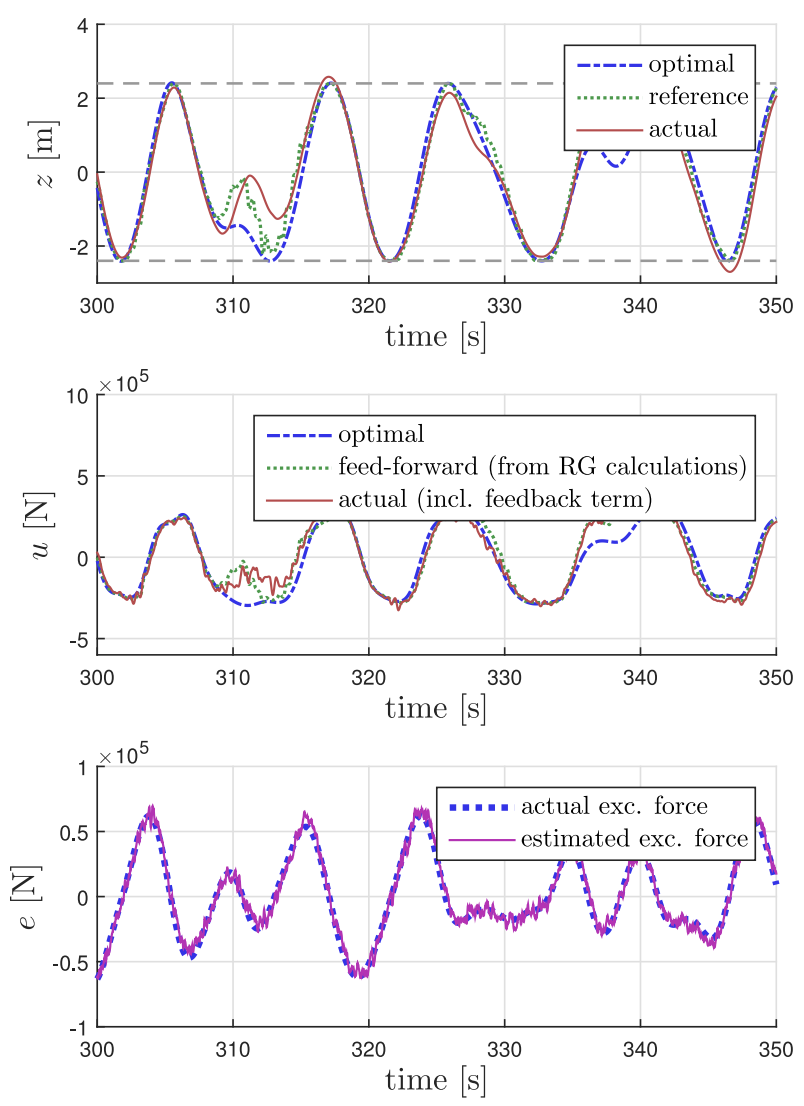

(b) Poles $p_{1}=p_{2}=-2$.

Fig. 14. Optimal, reference and actual WEC trajectory (top); optimal, non-linear feed-forward, and total control force (middle); actual and estimated excitation force (bottom).

and instead, setting the noise level proportionally to $\sigma_{x}$ (motivated by the fact that more measurement noise results in more errors in the excitation estimates) appears to be a reasonable choice. The recedinghorizon length is set to $T_{w}=3 T_{p}$.

Unsurprisingly, Fig. 15 shows that reactive control allows for a 2- to 4-fold increase over power absorption with respect to a simple linear damper, in the range of wave conditions considered. The recedinghorizon control configuration, regardless of whether the excitation force is perfectly known or has to be estimated from the WEC dynamics, results in sub-optimal power absorption, although generally well within $90 \%$ of the optimal. In the cases examined, the effect of measurement noise tends to be more pronounced for waves with longer periods (910). For shorter wave periods (6-8 s), there is very little difference in power absorption between the different receding-horizon variants C1C3. Consistently with previous studies (Auger et al., 0000; Li et al., 2012), the relatively poor WF performance (see Section 6) does not seem to significantly affect the results. This can be explained by the fact that the low-frequency components, which are crucial for wave energy absorption, are better predicted than their high-frequency counterparts.

For the cases considered here, the average computational time, necessary to solve each optimisation problem, is between 0.07 and $0.12 \mathrm{~s}$ depending on $T_{p}$, which is compatible with real-time implementation (below $\Delta T_{c}$ ).

To investigate the effects of TL inaccuracies, Fig. 16 provides, for the two noise levels considered, a comparison between the actual recedinghorizon results (solid lines), and those obtained assuming perfect tracking of the generated RT (dashed lines). The effect of TL imperfections is found to be relatively modest in terms of power absorption. However, as seen in Fig. 14b, tracking inaccuracies can result in small excursions outside the prescribed WEC constraints, which could need to be further addressed.

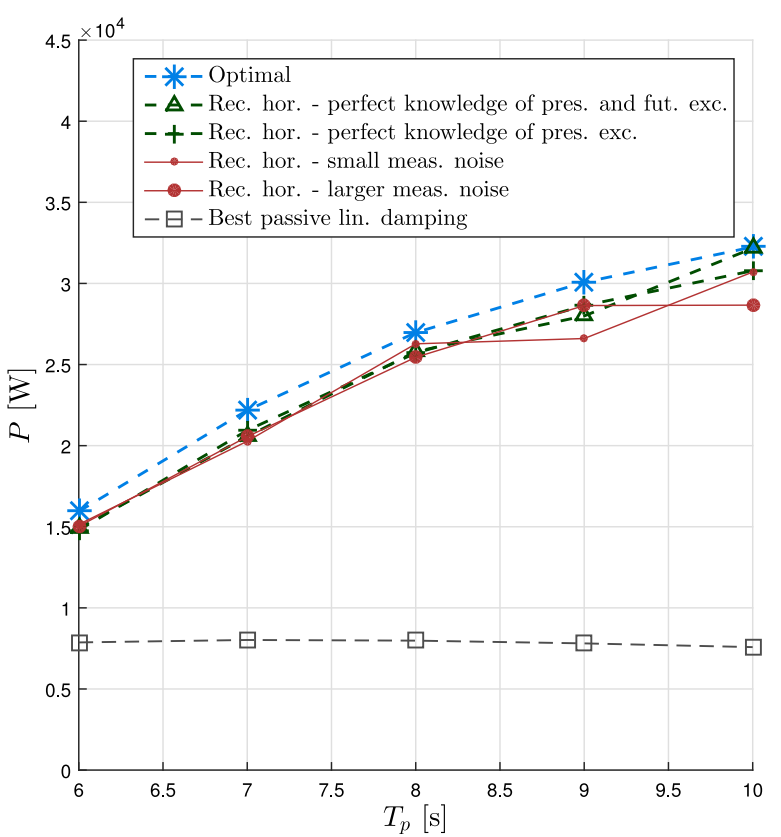

Fig. 15. Absorbed power with optimal control (computed off-line) and in various receding-horizon control configurations. A bi-directional power flow is allowed.

With uni-directional power flow

When passivity is required, RG computation is significantly more demanding. Furthermore, the RT resulting from the RG optimisation 


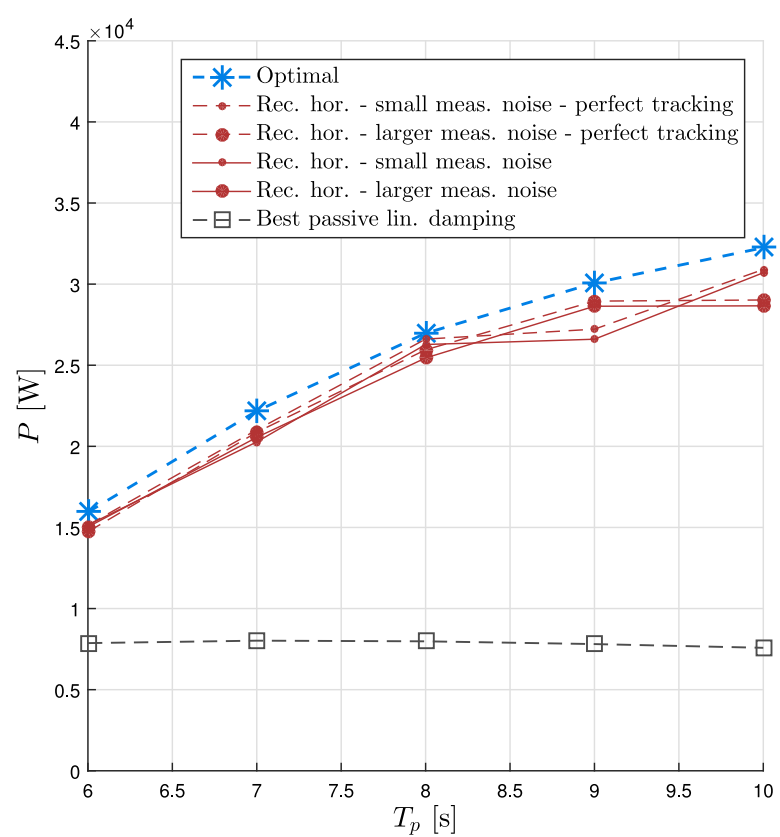

Fig. 16. Absorbed power with optimal control (computed off-line) and in different receding-horizon control configurations. A bi-directional power flow is allowed.
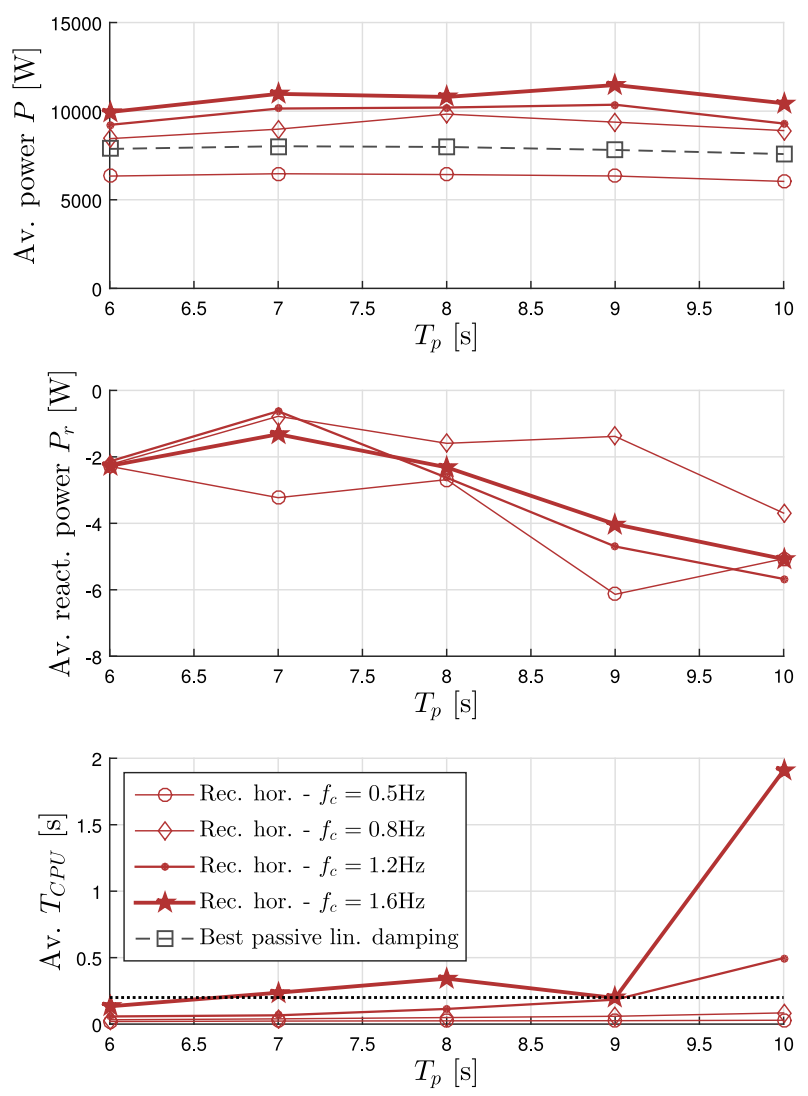

Fig. 17. Top graph: absorbed power in a receding-horizon control configuration (small meas. noise), with different cut-off frequencies $f_{c}$. Only a uni-directional power flow is allowed. Middle graph: average reactive power resulting from constraint violation. Bottom graph: average RG computation time ( $\Delta T_{c}$ is indicated through a dotted line).

may not ensure passivity between the constraint collocation points. Furthermore, increasing the number of collocation points also increases the control problem size, and therefore is detrimental to the computational performance of RG calculations. In addition, noise and imperfect
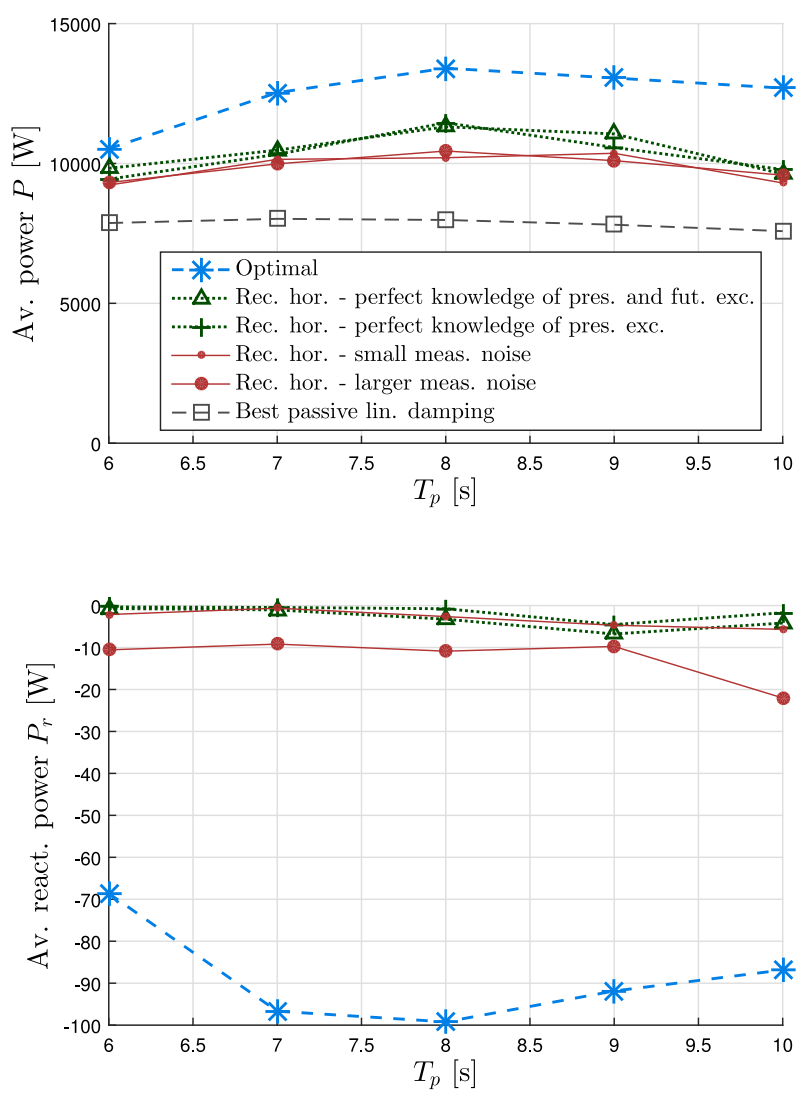

Fig. 18. Top graph: absorbed power with optimal control (computed off-line) and in various receding-horizon control configurations. Only a uni-directional power flow is allowed. Bottom graph: average reactive power resulting from constraint violation in the different configurations. Optimal calculation: $f_{c}=0.8 \mathrm{~Hz}$; rec. hor. calculations: $f_{c}=1.2$ Hz.

tracking may also lead to local violation of the passivity constraint. Therefore, the control force is modified in real time, so as to enforce passivity at the TL level, as follows:

$u^{\prime}=\left\{\begin{array}{l}u \text { if }-\dot{z}_{\text {est }} u>0 \\ 0 \text { if }-\dot{z}_{\text {est }} u \leq 0\end{array}\right.$

which is more compactly written as $u^{\prime}=\mathbf{1}_{\mathbb{R}^{+}}\left\{-\dot{z}_{\text {est }} u\right\} u$, where $\dot{z}_{\text {est }}$ is the estimated WEC velocity and $u=u_{\mathrm{fb}}+u_{\mathrm{ff}}$, as explained in Section 4 .

As mentioned earlier in Section 7.3, the receding window length is set to $T_{w}=2 T_{p}$. Fig. 17 shows the sensitivity of the receding-horizon control results to the cut-off frequency $f_{c}$ of the RG calculations, in terms of absorbed power $P$, passivity constraint violation (measured as $P_{r}$ ), and average RG calculation time. The measurement noise level is assumed small $\left(\sigma_{z}=0.025, \sigma_{\ddot{z}}=0.1\right)$. The simulation time is $T_{\text {sim }}=180$ s. If $f_{c}$ is set too low (e.g. $0.5 \mathrm{~Hz}$ ), the receding-horizon controller does not outperform a simple linear damper. Significant improvements are obtained by increasing the number of harmonics: for $f_{c}=1.6 \mathrm{~Hz}$, the improvement in power absorption amounts to $30-50 \%$ of the best passive linear damper results. However, the average computation time for RG calculations increases substantially with the number of harmonics, and actually exceeds $\Delta T_{c}=0.2 \mathrm{~s}$ for $f_{c}=1.6 \mathrm{~Hz}$ (bottom graph). Therefore, for this case study, $f_{c}=1.2 \mathrm{~Hz}$ is found to be an appropriate compromise. Finally, note that $f_{c}$ has little influence on the effective violation of the passivity constraint (middle graph).

The top graph of Fig. 18 shows the average steady-state absorbed power, obtained in a wave excitation signal of $T_{\text {sim }}=180 \mathrm{~s}$, for the different configurations detailed in Section 7.2. For the receding-horizon variants $\mathrm{C} 1-\mathrm{C} 3, f_{c}$ is set to $1.2 \mathrm{~Hz}$, based on the results of Fig. 17. The 

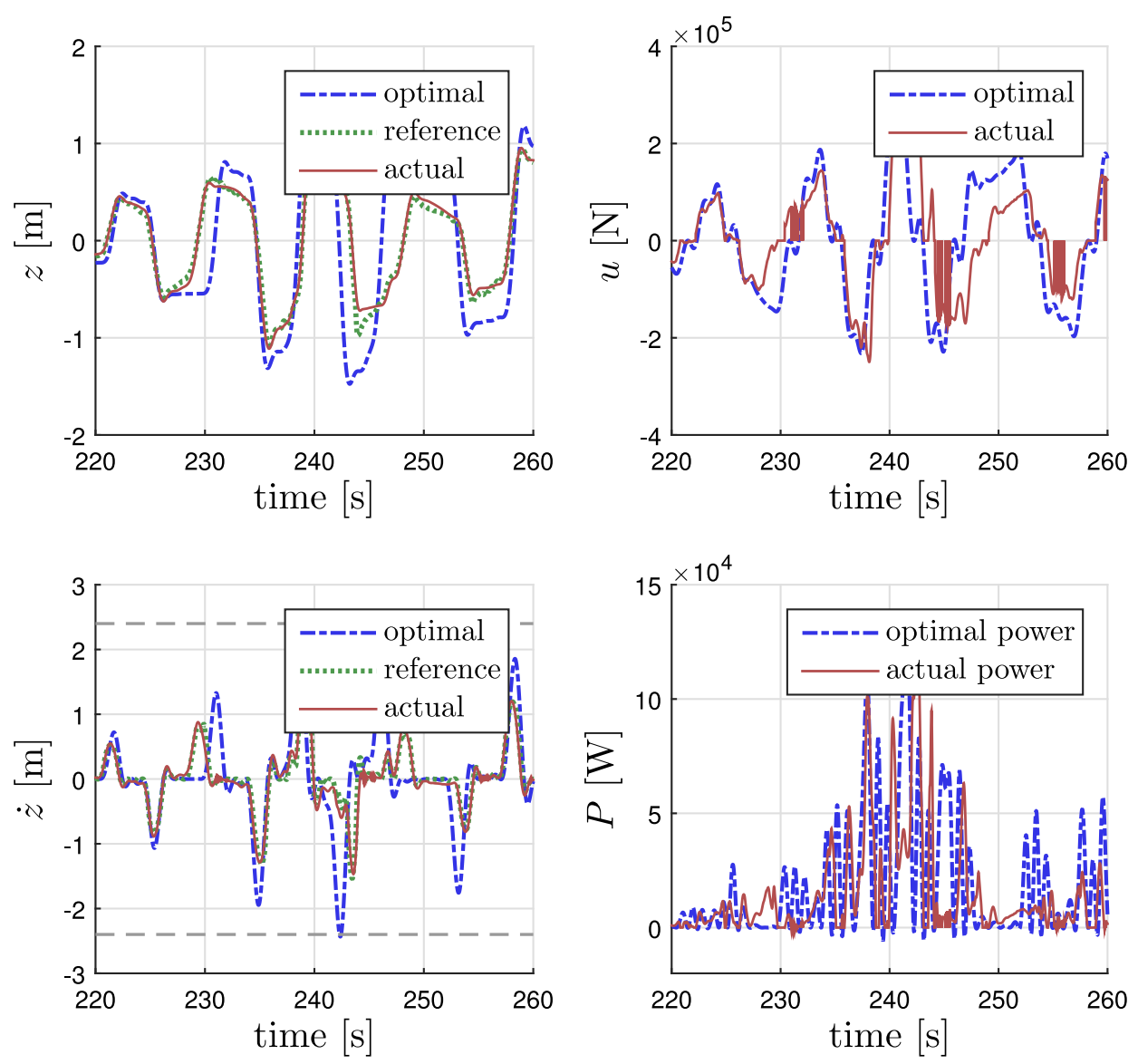

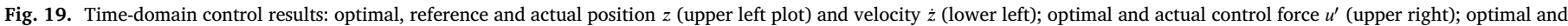
actual power absorption (lower right). JONSWAP spectrum $\left(H_{m_{0}}=1 \mathrm{~m}, T_{p}=9 \mathrm{~s}\right)$. Passivity enforcement as in Eq. (34).

bottom graph shows the average reactive power $P_{r}$, resulting from local violation of the passivity constraint.

Note that, in Fig. 18, no passivity enforcement is applied to the optimal control trajectory. Furthermore, while in this example the RG calculations are carried out with a cut-off frequency $f_{c}=1.2 \mathrm{~Hz}$, optimal calculations considering the $180 \mathrm{~s}$ wave signal in its entirety, with the same cut-off frequency, were not always achievable within a reasonable amount of time. Therefore, a cut-off frequency of $f_{c}=0.8 \mathrm{~Hz}$ is used instead. Optimal calculations, using the total wave signal, result in up to a $75 \%$ increase in absorbed power, with respect to the best passive damping. However, the passivity condition is not satisfied between collocation points, thus resulting in some reactive power being provided to the WEC, as measured by $P_{r}$ in the bottom graph. For the optimal control results computed off-line, $P_{r}$ is of the order of $1 \%$ of the average absorbed power $P$, which can be considered reasonably small: as a point of comparison, when reactive power is allowed (Section 8.3), $P_{r}$ is of the order of twice the value of $P$.

Results obtained in a receding-horizon configuration, with $f_{c}=$ $1.2 \mathrm{~Hz}$, are less favourable, in terms of absorbed power, than the optimal results computed off-line. However, passivity is successfully enforced at the TL level, reducing $P_{r}$ by a factor of more than 10 , with respect to off-line computation. The presence of measurement noise is observed to have a significant effect, on both average absorbed and reactive power. More specifically, higher measurement noise also causes greater error in $\dot{z}_{\text {est }}$, which, given the form of Eq. (34), makes passivity enforcement less accurate. Overall, the gains obtained in the recedinghorizon configurations of Fig. 18, with respect to a simple passive linear damper, are of the order of $20-30 \%$.

Finally, Figs. 19 and 20 examine passive control results in the time domain. In Fig. 19, obtained for a JONSWAP spectrum with
$H_{m_{0}}=1 \mathrm{~m}$ and $T_{p}=9 \mathrm{~s}$, the actual WEC trajectory is compared with the reference generated by the RG algorithm, and with the optimal trajectory computed off-line. As in Fig. 18, the comparison with the optimal trajectory has limited validity, because the cut-off frequency for the optimal calculation is $f_{c}=0.8 \mathrm{~Hz}, v s . f_{c}=1.2 \mathrm{~Hz}$ for the recedinghorizon calculations, and because the optimal trajectory yields more reactive power. It can be noted, however, how starkly the RT in Fig. 19 differs from that computed off-line.

Although passivity enforcement via Eq. (34) practically eliminates any reactive power flow, while still allowing for acceptable tracking of the RT, the resulting control force $u^{\prime}$ is, at times, subject to sharp transition - which is not dissimilar, in terms of control input requirements, to the bang-bang type control obtained in some other WEC control studies (for example Li et al., 2012).

However, it may be desirable to require a smoother control force. Therefore, it is suggested here to replace the indicator function $\mathbf{1}_{\mathbf{R}^{+}}$of Eq. (34) with a smooth approximation using a hyperbolic tangent, so that:

$u^{\prime}=\frac{1}{2}\left[\tanh \left(-k \dot{z}_{\mathrm{est}} u\right)+1\right] u$

where $k$ is some proportionality coefficient tuned, in this specific example, to $k=0.001$. The resulting trajectory, control force and power outputs can be seen in Fig. 20. While reactive power is also practically eliminated, the actual control force is now significantly smoother.

\section{Conclusions}

The proposed control framework contains four important components of a receding-horizon control architecture, namely reference trajectory calculations, tracking loop, estimation and forecasting. The 

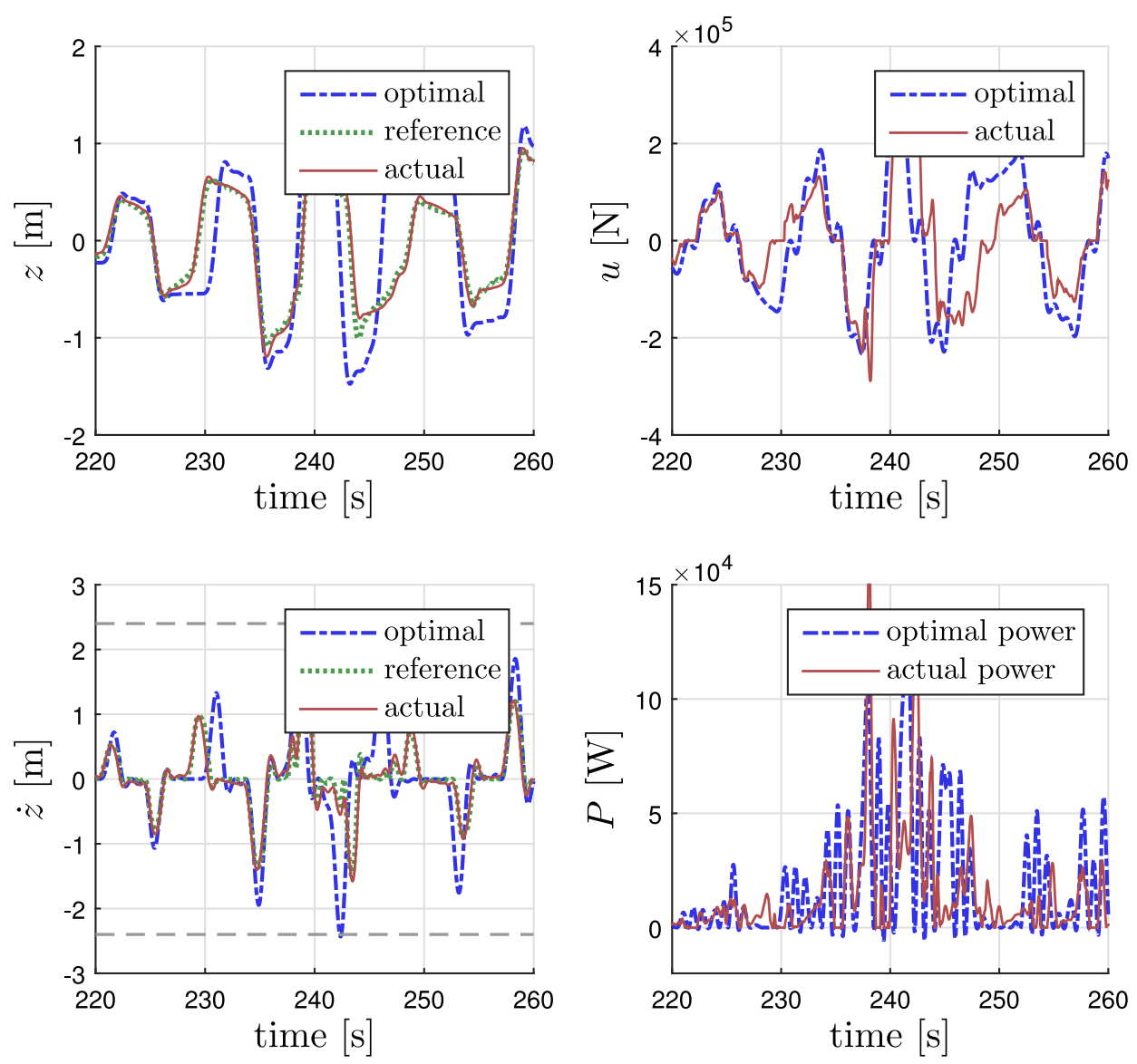

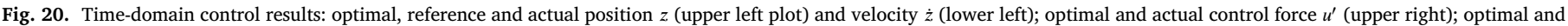
actual power absorption (lower right). JONSWAP spectrum $\left(H_{m_{0}}=1 \mathrm{~m}, T_{p}=9 \mathrm{~s}\right)$. Passivity enforcement as in Eq. (35).

overall controller performance, as well as the interplay between the four control components, are examined, based on a simple WEC model including a combination of static and dynamic non-linear forces, under constrained configurations, and taking into account imperfect measurement of the WEC dynamics. Furthermore, where possible, optimal control calculations, taking into account the complete input wave signal, are provided as a point of comparison, as well as the results from an optimally-tuned passive, constant linear damper.

RG calculations using a Fourier spectral method prove to be computationally attractive, and are successfully adapted to a receding-horizon framework. The TL, consisting of a combination of a feedback term with a non-linear feed-forward control force, allows for reasonably accurate trajectory tracking. The estimation and forecast errors, obtained respectively through the extended Kalman filter and the SBP method, do not seem to strongly affect the controller performance.

Strong interactions can be observed between the different components of the control structure. In particular, the dynamics of the RT, resulting not only from the RG optimisation, but also from the articulation of consecutive updates, should be taken into account when designing the TL and WE.

Overall, when reactive power flow is allowed, the receding-horizon framework allows for power absorption of the order of $90 \%$ of the optimal (and 2-4 times the best linear damper results), even with measurement noise.

When passivity is required, obtaining an 'optimal' point of comparison is significantly more complicated, mainly due to the high computational demand associated with passive control optimisation, over the whole simulation duration. However, the receding-horizon controller seems able to improve the average absorbed power significantly - of the order of $20-40 \%$ - with respect to the best linear damper, while being real-time compatible in a Matlab implementation. Even better results may be achievable by increasing the number of harmonics in the Fourier spectral RG calculations. This, however, could also unreasonably increase the computational requirements of the RG calculations. Therefore, it might be interesting to investigate the use of different, possibly discontinuous, basis functions (see for example Henriques, Lemos, Eça, Gato, \& Falcão, 2017).

In view of the results presented in this paper, further work and research orientations can be suggested:

- The quality of the RT could be improved, in particular regarding the transition between consecutive updates. In particular, it should be ensured that high-frequency dynamics, which unavoidably arise from a discrete sequence of trajectory updates, do not interfere with the TL. In addition, concerning more specifically the case with a passivity constraint, a more appropriate set of basis functions could perhaps yield better results.

- In turn, an improved quality of the RT should ease the task of the TL.

- The aforementioned issues, due to the discrete updates of the RT, would be strongly mitigated if more accurate WE and WF were achievable, because less frequent updates would be necessary, and because there would be less difference between two successive updates. This work, however, suggests that such improvements are only achievable by incorporating more measurements, such as wave elevation in the vicinity of the WEC, or pressure sensors, into the estimation and forecasting algorithms.

- The WE method, presented in this paper, relies heavily on an accurate description of the (non-linear) hydrodynamic model in the state-space description. The design of a WE, robust to modelling errors, could be the subject of further study. 


\section{Acknowledgement}

This paper is based upon work supported by Science Foundation Ireland under Grant No. 12/RC/2302 for the Marine Renewable Ireland (MaREI) centre.

\section{References}

Abdelkhalik, O., Zou, S., Bacelli, G., Robinett, R. D., Wilson, D. G., \& Coe, R. G. (2016). Estimation of excitation force on wave energy converters using pressure measurements for feedback control. In OCEANS 2016 MTS/IEEE Monterey (pp. 1-6). IEEE.

Andersen, P., Pedersen, T. S., Nielsen, K. M., \& Vidal, E. (2015). Model predictive control of a wave energy converter, 1540-1545.

Auger, C., Mérigaud, A., \& Ringwood, J. V. Receding-horizon pseudo-spectral control of wave energy converters using periodic basis functions, IEEE Transactions on Sustainable Energy.

Bacelli, G., Genest, R., \& Ringwood, J. V. (2015). Nonlinear control of flap-type wave energy converter with a non-ideal power take-off system. Annual Reviews in Control, 40, 116-126.

Bacelli, G., \& Ringwood, J. V. (2014). Nonlinear optimal wave energy converter control with application to a flap-type device. In Proceedings of the 19th IFAC world congress, vol. 47 (pp. 7696-7701). Elsevier.

Bazaraa, M. S., Sherali, H. D., \& Shetty, C. M. (2013). Nonlinear programming: theory and algorithms. John Wiley \& Sons.

Boyd, J. P. (2001). Chebyshev and Fourier spectral methods. Courier Corporation.

Brekken, T. K. (2011). On model predictive control for a point absorber wave energy converter. In PowerTech, 2011 IEEE Trondheim (pp. 1-8). IEEE.

Cavaglieri, D., Bewley, T. R., \& Previsic, M. (2015). Model predictive control leveraging ensemble kalman forecasting for optimal power take-off in wave energy conversion systems. In American Control Conference (ACC), 2015 (pp. 5224-5230). IEEE.

Cummins, W. (1962). The impulse response function and ship motions, Tech. rep. DTIC Document.

Davidson, J., Genest, R., \& Ringwood, J. V. (2017). Adaptive control of a wave energy converter simulated in a numerical wave tank. In Proceedings of the EWTEC 2017, Cork, 747.1-747.10.

Eaton, M. L. (2007). Multivariate statistics: A vector space approach. Lecture NotesMonograph Series, 53, i-512, URL http://www.jstor.org/stable/20461449.

Faedo, N., Olaya, S., \& Ringwood, J. V. (2017). Optimal control, MPC and MPC-like algorithms for wave energy systems: An overview. IFAC Journal of Systems and Control, 1, 37-56.

Faedo, N., Peña-Sanchez, Y., \& Ringwood, J. V. (2018). Finite-order hydrodynamic model determination for wave energy applications using moment-matching. Ocean Engineering, submitted for publication.

Falnes, J. (2002). Ocean waves and oscillating systems: linear interactions including waveenergy extraction. Cambridge University Press.

Fusco, F., \& Ringwood, J. V. (2010). Short-term wave forecasting for real-time control of wave energy converters. IEEE Transactions on Sustainable Energy, 1(2), 99-106.

Fusco, F., \& Ringwood, J. V. (2014). Hierarchical robust control of oscillating wave energy converters with uncertain dynamics. IEEE Transactions on Sustainable Energy, 5(3), 958-966. http://dx.doi.org/10.1109/tste.2014.2313479.

Genest, R., \& Ringwood, J. V. (2017). Receding horizon pseudospectral control for energy maximization with application to wave energy devices. IEEE Transactions on Control Systems Technology, 25(1), 29-38.

Gieske, P. (2007). Model predictive control of a wave energ converter: Archimedes wave swing. Master's thesis.

Guo, B., Patton, R., \& Jin, S. Identification and validation of excitation force for a heaving point absorber wave energy convertor. In Proceedings of the 12th European wave and tidal energy conference, 27th Aug. - 1st Sep. 2017, Cork, 2017.
Hals, J., Falnes, J., \& Moan, T. (2011). Constrained optimal control of a heaving buoy wave-energy converter. Journal of Offshore Mechanics and Arctic Engineering, 133(1), 011401.

Harris, F. J. (1978). On the use of windows for harmonic analysis with the discrete fourier transform. Proceedings of the IEEE, 66(1), 51-83.

Hasselmann, K., Barnett, T., Bouws, E., Carlson, H., Cartwright, D., Enke, K., et al. (1973). Measurements of wind-wave growth and swell decay during the joint north sea wave project (jonswap), Tech. rep. Deutches Hydrographisches Institut.

Henriques, J. C., Lemos, J. M., Eça, L., Gato, L. M., \& Falcão, A. F. (2017). A high-order discontinuous galerkin method with mesh refinement for optimal control. Automatica, $85,70-82$.

Li, G. (2015). Predictive control of a wave energy converter with wave prediction using differential flatness. In Decision and Control (CDC), 2015 IEEE 54th annual conference on (pp. 3230-3235). IEEE.

Li, G., Weiss, G., Mueller, M., Townley, S., \& Belmont, M. R. (2012). Wave energy converter control by wave prediction and dynamic programming. Renewable Energy, 48, 392403.

Ling, B. A. (2015). Real-time estimation and prediction of wave excitation forces for wave energy control applications. (Master's thesis), Oregon State University.

Mérigaud, A., \& Ringwood, J. V. Improving the computational performance of non-linear pseudo-spectral control of wave energy converters, IEEE Transactions on Sustainable Energy..

Mérigaud, A., \& Ringwood, J. V. Incorporating ocean wave spectrum information in shortterm free-surface elevation forecasting.

Mérigaud, A., \& Ringwood, J. V. Power production assessment for wave energy converters: Overcoming the perils of the power matrix, Proceedings of the Institution of Mechanical Engineers, Part M: Journal of Engineering for the Maritime Environment.

Mérigaud, A., \& Ringwood, J. V. (2017). Optimal trajectories, nonlinear models and constraints in wave energy device control. In Proceedings of the 20th IFAC world congress 2017 Toulouse, France.

Nielsen, S. R., Zhou, Q., Kramer, M. M., Basu, B., \& Zhang, Z. (2013). Optimal control of nonlinear wave energy point converters. Ocean Engineering, 72, 176-187.

Ochi, M. K. (2005). Ocean waves: The stochastic approach. Cambridge University Press.

Papoulis, A., \& Pillai, S. U. (2002). Probability, random variables, and stochastic processes. Tata McGraw-Hill Education.

Peña-Sanchez, Y., Garcia-Abril, M., Paparella, F., \& Ringwood, J. V. (2018). Estimation and forecasting of excitation force for arrays of wave energy devices PP (99) 1-1. http://dx.doi.org/10.1109/TSTE.2018.2807880.

Penalba Retes, M., Mérigaud, A., Gilloteaux, J. -C., \& Ringwood, J. (2015). Nonlinear froude-krylov force modelling for two heaving wave energy point absorbers. In Proceedings of the 11th European wave and tidal energy conference, European Wave and Tidal energy conference 2015.

Perez, T., \& Fossen, T. I. (2009). A matlab toolbox for parametric identification of radiation-force models of ships and offshore structures. Modeling, Identification and Control, 30(1), 1.

Richalet, J., Lavielle, G., \& Mallet, J. (2004). La commande prédictive: Mise en oeuvre et applications industrielles. Eyrolles.

Richter, M., Magana, M. E., Sawodny, O., \& Brekken, T. K. (2013). Nonlinear model predictive control of a point absorber wave energy converter. IEEE Transactions on Sustainable Energy, 4(1), 118-126.

Ringwood, J. V., Bacelli, G., \& Fusco, F. (2014). Energy-maximizing control of waveenergy converters: the development of control system technology to optimize their operation. IEEE Control Systems, 34(5), 30-55.

Scruggs, J., Lattanzio, S., Taflanidis, A., \& Cassidy, I. (2013). Optimal causal control of a wave energy converter in a random sea. Applied Ocean Research, 42, 1-15.

Tona, P., Nguyen, H. -N., Sabiron, G., \& Creff, Y. (2015). An efficiency-aware model predictive control strategy for a heaving buoy wave energy converter. In 11th European wave and tidal energy conference-EWTEC 2015.

de la Villa Jaén, A., Santana, A. G., et al. (2014). Considering linear generator copper losses on model predictive control for a point absorber wave energy converter. Energy Conversion and Management, 78, 173-183. 